\title{
The spatially resolved star formation history of mergers
}

\section{A comparative study of the LIRGs IC 1623, NGC 6090, NGC 2623, and Mice}

\author{
C. Cortijo-Ferrero ${ }^{1}$, R. M. González Delgado ${ }^{1}$, E. Pérez ${ }^{1}$, R. Cid Fernandes ${ }^{2}$, R. García-Benito ${ }^{1}$, P. Di Matteo ${ }^{3}$, \\ S. F. Sánchez ${ }^{4}$, A. L. de Amorim ${ }^{2}$, E. A. D. Lacerda ${ }^{2}$, R. López Fernández ${ }^{1}$, and C. Tadhunter ${ }^{5}$
}

\author{
1 Instituto de Astrofísica de Andalucía (CSIC), PO Box 3004, 18080 Granada, Spain \\ e-mail: clara@iaa.es \\ 2 Departamento de Física, Universidade Federal de Santa Catarina, PO Box 476, 88040-900 Florianópolis, SC, Brazil \\ 3 GEPI, Observatoire de Paris, PSL Research University, CNRS, Place Jules Janssen, 92190 Meudon, France \\ ${ }^{4}$ Instituto de Astronomía, Universidad Nacional Autonóma de México, AP 70-264, 04510 México DF, Mexico \\ 5 Department of Physics and Astronomy, University of Sheffield, Sheffield S3 7RH, UK
}

Received 22 May 2017 / Accepted 17 July 2017

\begin{abstract}
This paper presents the spatially resolved star formation history (2D-SFH) of a small sample of four local mergers: the early-stage mergers IC 1623, NGC 6090, and the Mice, and the more advanced merger NGC 2623, by analyzing IFS data from the CALIFA survey and PMAS in LArr mode. Full spectral fitting techniques are applied to the datacubes to obtain the spatially resolved mass growth histories, the time evolution of the star formation rate intensity $\left(\Sigma_{\mathrm{SFR}}\right)$, and the local specific star formation rate (sSFR), over three different time scales (30 Myr, $300 \mathrm{Myr}$, and $1 \mathrm{Gyr}$ ). The results are compared with non-interacting Sbc-Sc galaxies, to quantify if there is an enhancement of the star formation and to trace its time scale and spatial extent. Our results for the three LIRGs (IC $1623 \mathrm{~W}$, NGC 6090, and NGC 2623) show that a major phase of star formation is occurring in time scales of $10^{7} \mathrm{yr}$ to few $10^{8} \mathrm{yr}$, with global SFR enhancements of between approximately two and six with respect to main-sequence star forming (MSSF) galaxies. In the two early-stage mergers IC $1623 \mathrm{~W}$ and NGC 6090, which are between first pericentre passage and coalescence, the most remarkable increase of the SFR with respect to non-interacting spirals occurred in the last $30 \mathrm{Myr}$, and it is spatially extended, with enhancements of factors between two and seven both in the centres $(r<0.5$ half light radius, HLR), and in the disks $(r>1$ HLR). In the more advanced merger NGC 2623 an extended phase of star formation occurred on a longer time scale of $\sim 1$ Gyr, with a SFR enhancement of a factor of approximately two-to-three larger than the one in Sbc-Sc MSSF galaxies over the same period, probably relic of the first pericentre passage epoch. A SFR enhancement in the last $30 \mathrm{Myr}$ is also present, but only in NGC 2623 centre, by a factor of three. In general, the spatially resolved SFHs of the LIRG-mergers are consistent with the predictions from high spatial resolution simulations. In contrast, the star formation in the Mice, specially in Mice B, is not enhanced but inhibited with respect to Sbc-Sc MSSF galaxies. The fact that the gas fraction of Mice B is smaller than in most non-interacting spirals, and that the Mice are close to a prograde orbit, represents a new challenge for the models, which must cover a larger space of parameters in terms of the availability of gas and the orbital characteristics.
\end{abstract}

Key words. techniques: imaging spectroscopy - galaxies: evolution - galaxies: stellar content - galaxies: interactions

\section{Introduction}

Galaxy interactions, and in particular mergers, enhance the star formation, leading to temporarily increased star formation rates (SFRs), sometimes by large amounts. However, even if triggering occurs, the observability time scale of the burst may be shorter than the duration of the interaction. In other words, a galaxy encounter may not be recognized if observed long after the peak of activity. Mergers of gas rich disks, such as those seen in luminous and ultraluminous infrared galaxies (LIRGs and ULIRGs; Surace et al. 1998, 2000; Veilleux et al. 2002; Kim et al. 2013) are extreme systems, where we directly observe the merger-induced star formation (Sanders \& Mirabel 1996). Given their IR luminosities, they could reach SFRs as high as $\sim 170 M_{\odot} \mathrm{yr}^{-1}$, according to the relation between $L_{\mathrm{FIR}}$ and SFR provided by Kennicutt (1998). However, on average, the SFR increase in mergers (not necessarily reaching U/LIRG luminosities) is more modest: a factor of a few with respect to control samples of non-interacting galaxies (Barrera-Ballesteros et al. 2015; Knapen et al. 2015). This limited SFR enhancement is also predicted by numerical modelling (Di Matteo et al. 2007, 2008; Moreno et al. 2015). Di Matteo et al. (2007) found that the starbursts induced by major mergers have a moderate intensity, with SFRs rarely being enhanced by factors larger than five compared to isolated galaxies, even at the peak of the starbursts. However, about 15\% of galaxy interactions models have a merger-triggered starburst with a relative enhancement higher than five, in agreement with the vast majority of LIRGs and ULIRGs in the local universe. Recent work (both theoretical and observational) has shown that minor mergers also produce SFR enhancements of a few, that are similar to those produced by major mergers, and even a factor of ten or more in the most extreme cases (Kaviraj 2014; Starkenburg et al. 2016; Hernquist 1989; Mihos \& Hernquist 1994).

The spatial scale of the star formation is another important factor that must be taken into account when comparing the star formation in mergers with non interacting control galaxies. Low resolution merger simulations that parameterize the SFR as a function of the local gas density (Kennicutt-Schmidt law), and that do not treat gas fragmentation, predict that most star 
formation is strongly concentrated towards the central region of the merger remnant, and even suppressed in the outskirts (Mihos \& Hernquist 1996; Moreno et al. 2015). These models underestimate the spatially extended star formation observed in many galaxy mergers from early-stage merger or separated progenitors (Wang et al. 2004; Elmegreen et al. 2006) to advanced or post coalesced systems (Evans et al. 2008; Wilson et al. 2006). Recently, Smith et al. (2016) compared the SFRs of 700 star forming complexes in 46 nearby interacting galaxy pairs (only early-stage mergers) with those in 39 normal spiral galaxies. They found that the regions with highest SFRs in the interacting systems lie at the intersection of spiral or tidal structures. There exists an excess of high SFR clumps in interacting galaxies which is not observed in normal spirals.

Barnes (2004) shows that SF is extended, when, instead of adopting a purely density dependent SF prescription, energy dissipation in shocks is also taken into account. New high resolution models in the literature that determine the SFR efficiency in mergers by resolving parsec scale physical processes (Teyssier et al. 2010; Hopkins et al. 2013; Renaud et al. 2015, 2016) find that extended starbursts arise spontaneously (without any SFR prescription) due to fragmentation of the gas clouds produced by the increase of the supersonic turbulence of the interstellar medium (ISM) as a consequence of the tidal interaction itself. In these models, a merger-induced nuclear starburst is also present, but it occurs later in the merger sequence. They conclude that the extended star formation is important in the early stages of the merger, while nuclear starbursts will occur in the advanced stages. Hopkins et al. (2013) note the importance of properly treating the stellar feedback in numerical models resolving the formation of dense clouds, as predictions critically depend on the physics that may (or may not) destroy or disperse those clouds.

Most stars in the local Universe and up to redshift $z \sim 2$, are not formed in mergers, but in normal star forming galaxies along the so-called main sequence, as revealed both by observations (Lofthouse et al. 2017; Rodighiero et al. 2011; Wolf et al. 2005), and simulations (Fensch et al. 2017; Dekel et al. 2009). However, mergers are still a crucial phase towards the quenching of star formation and morphological transformation in galaxies. In that sense, local U/LIRGs are unique laboratories offering insight into the physical processes triggered by galaxy mergers. Moreover, the observational characterization of star formation in mergers at different stages is necessary to test the validity and to constrain merger simulations. Current integral field spectroscopy (IFS) surveys are very promising for studying merger evolution, as they offer spatially resolved information.

The goal of this paper is to obtain the spatially resolved star formation history (SFH) of mergers to determine if there is enhanced star formation, its time scale, and the spatial extent of these bursts of star formation. Here the study is limited to a small sample of local mergers: three early stage mergers (Mice, IC 1623, and NGC 6090) and a more advanced merger, NGC 2623. Except for the Mice, the other three systems are LIRGs. The four systems are representative cases of local mergers at distances of $\leq 127 \mathrm{Mpc}$, which correspond to a spatial sampling of $\leq 615 \mathrm{pc} / \mathrm{arcsec}$.

This paper is organized as follows: Sect. 2 describes the main properties of the galaxies analyzed here. Section 3 describes briefly the observations and data reduction. Section 4 summarizes our method for extracting the SFH, and the spatially resolved results. Section 5 deals with the radial profiles of the intensity of the star formation $\left(\Sigma_{\mathrm{SFR}}\right)$ and local specific star formation rate. In Sect. 6, we discuss the results in relation to the enhancement of star formation, its spatial extent and the time scale of the star formation in comparison with spiral galaxies. Section 7 reviews of our main findings.

\section{Sample}

Our study concentrates on four mergers: the Mice, IC 1623, NGC 6090, and NGC 2623. Except for IC 1623, the other systems are part of the CALIFA survey (Sánchez et al. 2012, 2016). These galaxies do not make a complete sample of interacting and merger systems of CALIFA, but they are already well characterized systems in terms of the spatially resolved properties, and they are proto-type of early-stage mergers (Mice, IC 1623, NGC 6090) and more advanced mergers (NGC 2623). Thus, they constitute a suitable sample for this pilot study that will be extended in the future for a larger CALIFA sub-sample. Based on IFS data, a detailed analysis on the spatially resolved stellar population properties for IC 1623 and NGC 6090 are presented in Cortijo-Ferrero et al. (2017a), for NGC 2623 in Cortijo-Ferrero et al. (2017b), and for the Mice in Wild et al. (2014). The main properties of the sample are summarized in Table 1. Also, a summary of the spatially resolved stellar population properties is:

- Mice: The stellar populations of both galaxies are predominantly older than several Gyrs. Only the northern tidal tail and the NE arm of NGC 4676B have distinctly younger stellar populations, with light-weighted ages of $\sim 0.6 \mathrm{Gyr}$. The fraction of stellar mass contributed by stars younger than $140 \mathrm{Myr}$ is less that 5\% in all regions, although $\sim 30 \%$ of optical light in the nuclear regions of NGC 4676A and tidal arm of NGC 4676B arises from stars younger than 140 Myr. There is an excess of light from intermediate age stars seen in the western half of NGC 4676A, inter-galaxy region, and NE bar of NGC 4676B, compared to the disks of these galaxies. This is consistent with the triggering of low level star formation in the gas flung out from the galaxy disks at first passage $\sim 170$ Myr ago, perhaps through dynamic instabilities or shocks. However, the ongoing merger has had little significant effect on the stellar populations in terms of total stellar mass or global star formation rate. In agreement with the observations, a standard hydrodynamical merger simulation of the Mice also shows little enhancement in star formation during and following first passage (Wild et al. 2014).

- IC 1623: Merger-induced star formation is extended and recent, as revealed by the widespread star clusters. The $\mathrm{W}$ progenitor has an average light-weighted age of $\sim 50 \mathrm{Myr}$. IC $1623 \mathrm{~W}$ centre $(<0.5 \mathrm{HLR})$ is older, $\sim 300 \mathrm{Myr}$, than the surrounding area, $30 \mathrm{Myr}$, due to the presence of a significant contribution of intermediate-age stellar populations, probably induced in a previous stage of the merger. However, the light is everywhere dominated by young stellar populations, with contributions much higher than the typical 10-20\% in Sbc/Sc control galaxies. The extinction in the $\mathrm{W}$ progenitor is $\sim 0.2 \mathrm{mag}$, and has a flat radial profile in comparison with $\mathrm{Sbc/Sc}$ control spirals. However, we note that IC $1623 \mathrm{E}$ is affected by much higher levels of extinction (up to 2-6 mag) according to the star cluster photometry. IC $1623 \mathrm{~W}$ has a mean stellar metallicity of $\sim 0.6 Z_{\odot}$, with a flat or slightly positive radial profile. The average $\operatorname{SFR}(t<30 \mathrm{Myr})$ of IC $1623 \mathrm{~W}$ is a factor of six above the SFR of main-sequence Sbc-Sc galaxies of the same mass (Cortijo-Ferrero et al. 2017a). 
Table 1. Sample properties.

\begin{tabular}{lcccc}
\hline \hline Property & Mice & IC 1623 & NGC 6090 & NGC 2623 \\
\hline CALIFA ID & $577(\mathrm{~A}) ; 939(\mathrm{~B})$ & - & 2945 & 213 \\
RA & 124610.7 & 010746.3 & 161140.8 & 083823.8 \\
Dec & +304338 & -173032 & +522727 & +254517 \\
Interaction stage & IIIa & IIIb & IIIb & IV \\
$z$ & 0.022049 & 0.020067 & 0.029304 & 0.018509 \\
Scale $\left(\mathrm{kpc} /{ }^{\prime \prime}\right)$ & 0.47 & 0.42 & 0.61 & 0.39 \\
HLR $(\mathrm{kpc})$ & $4.6(\mathrm{~A}) ; 3.8(\mathrm{~B})$ & 2.8 & 4.2 & 3.3 \\
Stellar mass $\left(M_{\odot}\right)$ & $1.2 \times 10^{11}(\mathrm{~A}), 1.5 \times 10^{11}(\mathrm{~B})$ & $3.9 \times 10^{10}$ & $6.8 \times 10^{10}$ & $5.4 \times 10^{10}$ \\
$\log \left(L_{\mathrm{IR}}\left[L_{\odot}\right]\right)$ & 10.62 & 11.65 & 11.51 & 11.54 \\
$S F R_{30 \mathrm{Myr}}\left(M_{\odot} / \mathrm{yr}\right)$ & $3(\mathrm{~A}), 2(\mathrm{~B})$ & 20 & 51 & 8 \\
\hline
\end{tabular}

Notes. Rows from top to bottom are: (1) CALIFA identification number, (2) and (3) right ascension and declination from Equinox J2000 extracted from Sanders et al. (2003), (4) interaction stage according to Veilleux et al. (2002) classification based on the optical morphologies, (5) redshift extracted from NED webpage, (6) spatial scale, (7) half light radius, (8) stellar masses extracted from Wild et al. (2014), Cortijo-Ferrero et al. (2017a,b), (9) infrared luminosities $L_{\mathrm{IR}}=L(8-1000) \mu \mathrm{m}$ from IRAS fluxes extracted from Sanders et al. (2003) for the LIRGs. They are in logarithm and in units of solar bolometric luminosity, $L_{\odot}=3.83 \times 10^{33} \mathrm{erg} \mathrm{s}^{-1}$. Mice $L_{\mathrm{IR}}$ is extracted from Wild et al. (2014) and (10) SFR calculated in the last $30 \mathrm{Myr}$, from the spectral synthesis in this paper.

- NGC 6090: Merger-induced widespread clusters are also present in this early-stage merger, with an average light-weighted age of the stellar populations 50-100 Myr. The age profiles are significantly flatter than in $\mathrm{Sbc} / \mathrm{Sc}$ galaxies, in agreement with a general rejuvenation of the progenitor galaxies due to merger-induced star formation. There exists a difference of $\sim 1$ mag between the stellar extinctions of the two progenitors, with an average of $1.3 \mathrm{mag}$ in NGC 6090 NE, and 0.5 mag in NGC 6090 SW, and whose radial profiles are flat in comparison with $\mathrm{Sbc/Sc}$ control spirals. NGC 6090 has a mean stellar metallicity of $\sim 0.6 Z_{\odot}$, with a flat or slightly positive radial profile. The average $\operatorname{SFR}(t<30 \mathrm{Myr})$ is increased by a factor of nine with respect to main-sequence Sbc-Sc galaxies. In this study we will better quantify the spatial extension and time scale of the SFR enhancement (Cortijo-Ferrero et al. 2017a).

- NGC 2623: There were two periods of merger-induced star formation in NGC 2623: a recent episode, traced by young stellar populations $(<140 \mathrm{Myr})$ located in the innermost $(<0.5 \mathrm{HLR} \sim 1.4 \mathrm{kpc})$ central regions, and in some isolated clusters in the northern tidal tail and in the star forming knots south of the nucleus; and a previous and widespread ( $\sim 2$ HLR $\sim 5.6 \mathrm{kpc})$ episode, traced by the spatially extended intermediate-age stellar populations with ages between $140 \mathrm{Myr}-1.4 \mathrm{Gyr}$. Due to this distribution of the stellar populations, the centre of NGC $2623(<0.2 \mathrm{HLR})$ is younger, $500 \mathrm{Myr}$ on average, than at $1 \mathrm{HLR}, \sim 900 \mathrm{Myr}$, and the age gradient is positive $(\sim 400 \mathrm{Myr})$, in contrast with the negative gradients in Sbc-Sc galaxies. The stellar extinction is high in the inner 0.2 HLR $(\sim 1.4 \mathrm{mag})$, and shows a negative gradient, which is much steeper $(-0.9 \mathrm{mag})$ than in Sbc-Sc galaxies, $-0.3 \mathrm{mag}$, indicating that in the last stages of a merger most of the gas and dust content of both progenitors is concentrated in the mass centre of the system (Cortijo-Ferrero et al. 2017b).

\section{Observations and data reduction}

The observations were carried out at Calar Alto observatory (CAHA) with the $3.5 \mathrm{~m}$ telescope and the Potsdam
Multi-Aperture Spectrometer (PMAS; Roth et al. 2005). For IC 1623 we used data with the Lens Array (LArr) configuration with a spatial magnification of $0.75^{\prime \prime} /$ lens, covering a $12^{\prime \prime} \times 12^{\prime \prime}$ field of view (FoV). Two pointings were taken to cover the whole western galaxy (see Cortijo-Ferrero et al. 2017a, for details). For the other systems, we use the PPaK mode (Verheijen et al. 2004) with a FoV of $74^{\prime \prime} \times 64^{\prime \prime}$, and 382 fibers of $2.7^{\prime \prime}$ diameter each (Kelz et al. 2006). IC 1623 was observed with the V300 grating and the other galaxies using a combination of the gratings V500 and V1200, named in CALIFA as COMBO mode. These different set ups, however, cover a similar wavelength range 23700-7100 $\AA$ and with a spectral resolution of FWHM $7 \AA$ for V300 and $\sim 6 \AA$ for COMBO data. While in the LArr data the spatial sampling is $0.75^{\prime \prime} /$ spaxel, for PPaK the final sampling is $1 \mathrm{arcsec} / \mathrm{spaxel}$.

Data of IC 1623 was calibrated as explained in Cortijo-Ferrero et al. (2017a) and the data for the other galaxies were calibrated with version V1.5 of the reduction pipeline (García-Benito et al. 2015). We refer to Sánchez et al. (2012), Husemann et al. (2013), García-Benito et al. (2015), and Sánchez et al. (2016) for details on the observational strategy and data processing of these data cubes.

In Fig. 1 we show the HTS/ACS (F814W) maps of the four galaxies in our sample. The positions of one and two half light radii (HLR) are indicated in the figure. Our radial distances will be expressed in units of HLR, this is defined as the semi-major axis length of the elliptical aperture which contains half of the total light of the galaxy at the rest-frame wavelength $5635 \AA$ (see Cid Fernandes et al. 2013; González Delgado et al. 2014, for details). In these galaxies, 1 HLR is $~ 4.6,3.8,2.8,4.2$, and $3.3 \mathrm{kpc}$ for the Mice A, Mice B, IC 1623 W, NGC 6090, and NGC 2623, respectively, on average, while for the Sbc and Sc control galaxies, $1 \mathrm{HLR} \sim 5.0 \mathrm{kpc}$, and $4.1 \mathrm{kpc}$, respectively.

\section{Spatially resolved star formation histories}

\subsection{Method of analysis}

LArr and PPaK datacubes were analyzed using the full spectral synthesis technique applied in a similar way as we have done in 

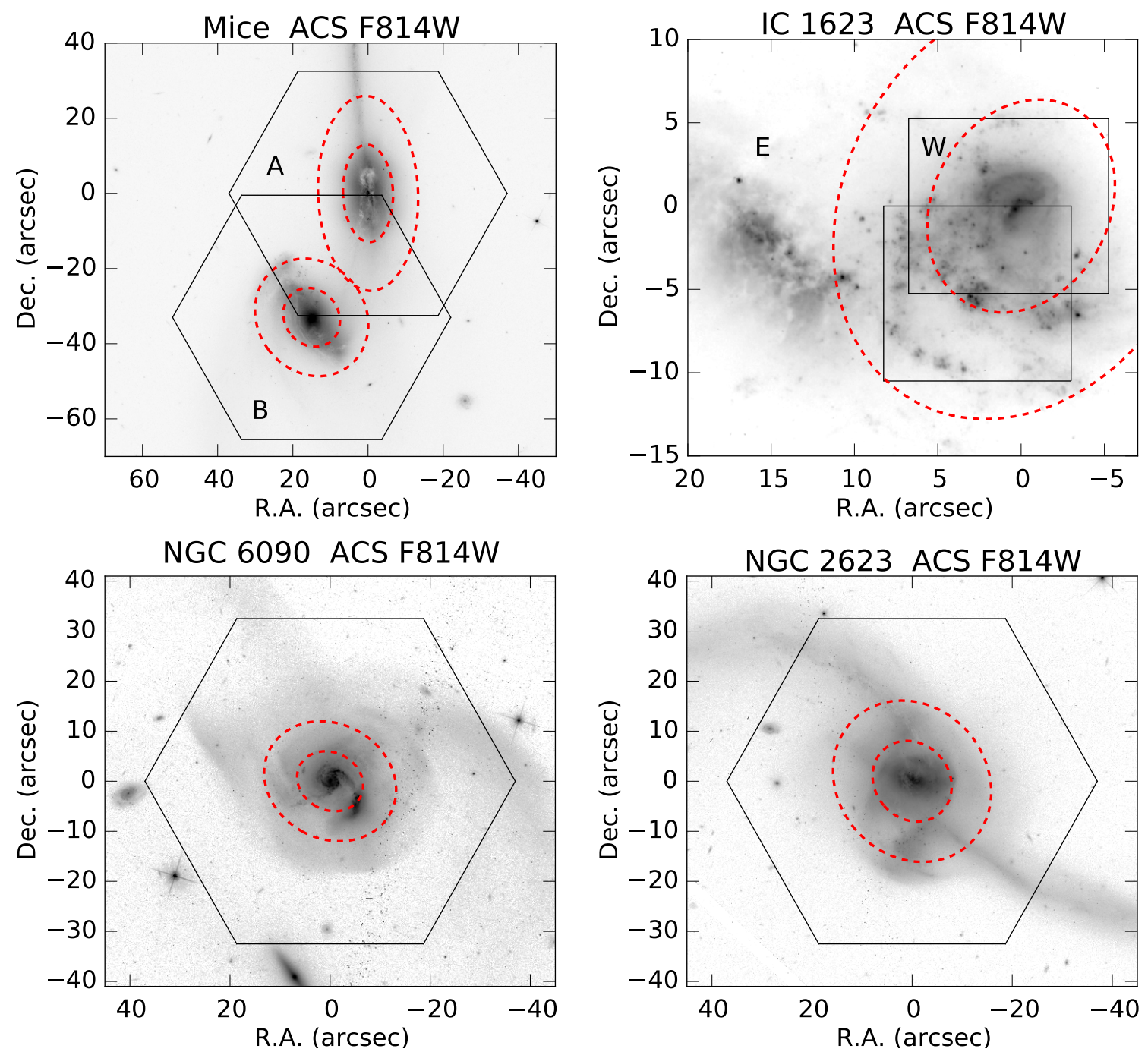

Fig. 1. HST/ACS (F814W) images of the four galaxies in our sample. The fields observed with IFS are shown by hexagons (PPaK) or squares (LArr). The red dashed circles indicate the position of 1 and 2 HLR centred at the nuclei of the main galaxy.

Pérez et al. (2013) and Cid Fernandes et al. (2013, 2014). The individual spectra were extracted from the CALIFA data by coadding spaxels into Voronoi zones (Cappellari \& Copin 2003) to get a $S / N>20$; from the LArr we used the individual spaxels if the $S / N>5$. To extract the star formation histories we used the STARLIGHT code (Cid Fernandes et al. 2005), which fits the observed spectra through a $\chi^{2}$ minimization, using a linear combination of simple stellar populations (SSPs) from a base of models spanning different ages and metallicities. For the SSPs we used a combination of the Vazdekis et al. (2010) for ages older than 63 Myr and González Delgado et al. (2005) for younger ages, formed by 156 spectra of 39 different ages from 1 Myr to $14 \mathrm{Gyr}^{1}$, and four metallicities $\left(Z=0.2,0.4,1\right.$, and $\left.1.6 Z_{\odot}\right)$. As in Cortijo-Ferrero et al. (2017a), dust effects were modelled as a foreground screen with a Calzetti et al. (2000) reddening law with $R_{V}=4.5$, and assuming the same reddening for all the SSP components. The output was then processed through PyCASSO (the Python CALIFA STARLIGHT Synthesis Organizer)

1 SSP ages are: $1 \mathrm{Myr}, 3,4,5.6,8.9,10,12.6,14.1,17.8,20,25.1$, $31.6,39.8,56.2,63,63.1,70.8,100,112,126159,200,282,355,501$, $708,891 \mathrm{Myr}$, and 1.1 Gyr, 1.3, 1.4, 2.0, 2.5, 3.5, 4.5, 6.3, 7.9, 10, 12.3, and $14.1 \mathrm{Gyr}$. to produce a suite of the spatially resolved stellar population properties.

As most U/LIRGs, and in particular, the systems in our paper, present a strong contribution of the stellar populations younger than 1 Gyr (Cortijo-Ferrero et al. 2017a,b; Alonso-Herrero et al. 2010; Pereira-Santaella et al. 2015), our non-parametric approach is the best to unveil their complex SFH. Other methods of analysis commonly used to predict the SFR of normal, non-interacting galaxies, like the parametric exponentially declining SFH fitting or $\tau$ models (Maraston et al. 2013, 2010) will not be able to predict correct and simultaneously the contribution of the young and/or the old stellar population.

Radial profiles of the age, metallicity, stellar mass surface density, and extinction are presented in Cortijo-Ferrero et al. (2017a) for IC 1623 and NGC 6090, and in Cortijo-Ferrero et al. (2017b) for NGC 2623. In the next sections, the results for spatially resolved SFH and radial profiles of the specific SFR and the intensity of the SFR (SFI) are presented and compared with similar results obtained for two subsamples of Sbc/Sc galaxies from the CALIFA mother sample that were analyzed in González Delgado et al. (2016, 2017). They were selected in the same mass range as the mergers, and are formed by $70 \mathrm{Sbc}$ and $14 \mathrm{Sc}$ galaxies. These late spirals are chosen for this comparison study because the progenitors of the galaxy mergers in 

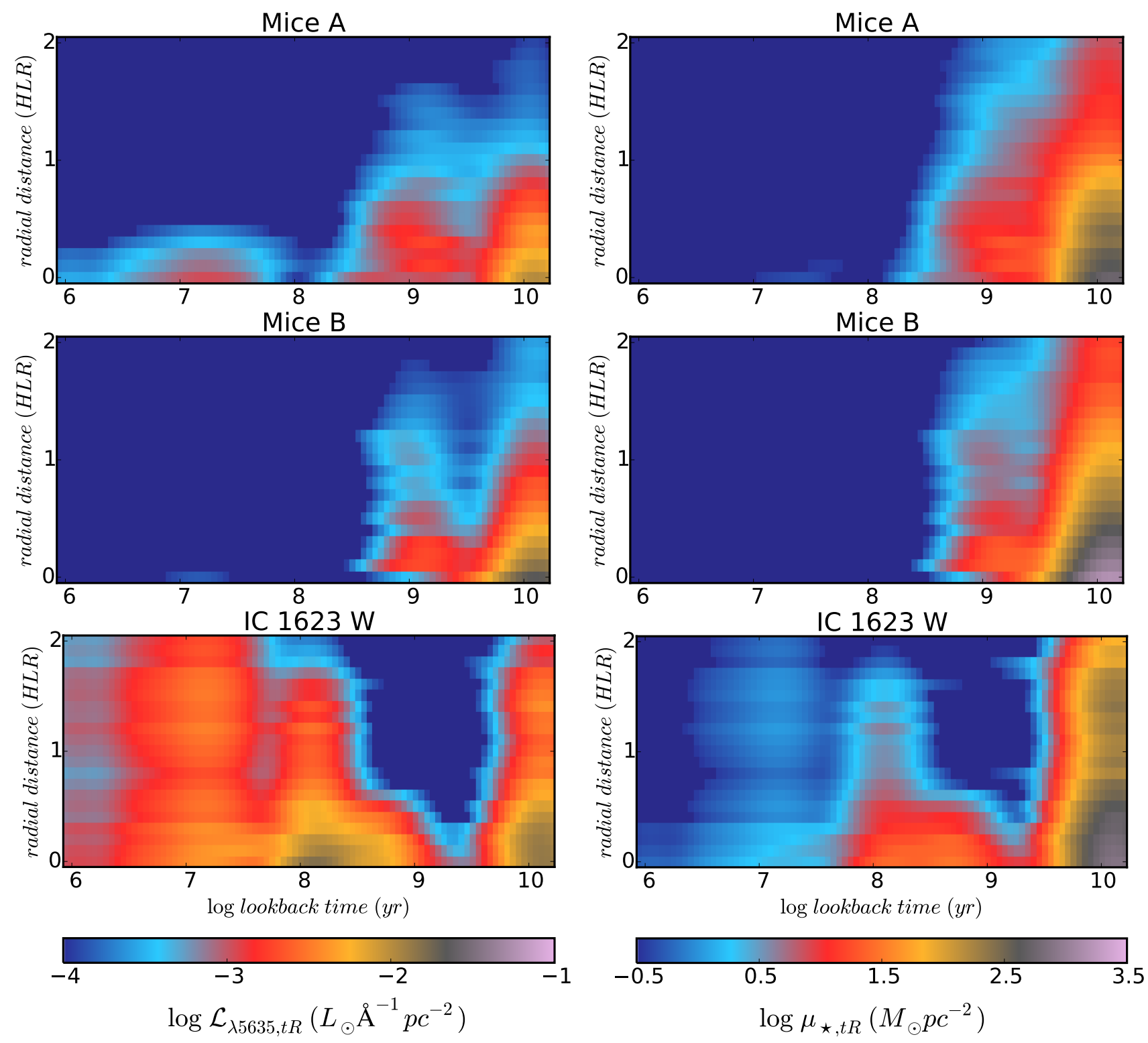

Fig. 2. $R \times t$ diagrams showing the radially averaged distribution of the mass and light as a function of the distance (in HLR units) from the nucleus and lookback time (in log units). The intensity of the map shows: stellar mass formed per unit area $\left(M_{\odot} \mathrm{pc}^{-2}\right.$; right panels), and luminosity at $5635 \AA$ per unit area $\left(L_{\odot} \AA^{-1} \mathrm{pc}^{-2}\right.$; left panels). From top to bottom: Mice A, Mice B, IC $1623 \mathrm{~W}$.

our sample are gas-rich spirals without significant bulges. This allows us to find out if there is an enhancement of the star formation with respect to non-interacting systems.

\section{2. $S F H$ : the $2 D R \times t$ maps}

As explained in Cid Fernandes et al. (2013) an interesting way to visualize the spatially resolved star formation history of a galaxy is the $R \times t$ diagrams. There, the $y$-axis is the radial distance $(R)$ and the $x$-axis is the lookback time $(t)$ and the intensity of the map is the luminosity density $\mathcal{L}_{\lambda 5635, t R}$ (corrected by extinction) or the mass surface density $\mu_{\star, t R}$. These maps represent the mass in stars per $\mathrm{pc}^{2}$ at each radial distance formed at each cosmic time epoch and the luminosity emitted by these stars. These $R \times t$ diagrams were obtained as follows. For each spectrum that corresponds to an $x y$ location in the galaxy, STARLIGHT provides the light fraction $\left(x_{x y t z}\right)$ at the normalized wavelength $(\lambda=5635 \AA$ as in previous studies) due to the base population with age $t$ and metallicity $Z$. Taking into account the stellar extinction and $M / L$ spatial variation, STARLIGHT also provides the mass fraction $\left(m_{x y t z}\right)$. Then, $x_{x y t z}$ and $m_{x y t Z}$ are collapsed in the $Z$ domain, and the $x y$ locations are compressed in an azimuthally average radial distance, to get the luminosity density, $\mathcal{L}_{\lambda 5635, t R}\left(L_{\odot} \AA^{-1} \mathrm{pc}^{-2}\right)$ and mass density $\mu_{\star, t R}$ at each radial position $R$ and for each age (equivalent to time evolution, expressed in lookback time).

The $R \times t$ diagrams of the galaxies are presented in Fig. 2, where the luminosity density $\mathcal{L}_{\lambda 5635, t R}\left(L_{\odot} \AA^{-1} \mathrm{pc}^{-2}\right)$ and mass density $\mu_{\star, t R}\left(M_{\odot} \mathrm{pc}^{-2}\right)$ are on the left and right panels, respectively. For the purpose of visualization, the original $\mathcal{L}_{\lambda 5635, t R}$ and $\mu_{\star, t R}$ maps $^{2}$ are smoothed in $\log t$, applying a Gaussian filter with $F W H M=0.5$ dex. For comparison, the $\mathcal{L}_{\lambda 5635, t R}$ and $\mu_{\star, t R}$ maps of the Sbc/Sc control non-interacting spirals are also

2 Due to the lack of temporal resolution, for ages $<30$ Myr we assign an average value of the mass and luminosity densities using all the SSPs younger than that age. 

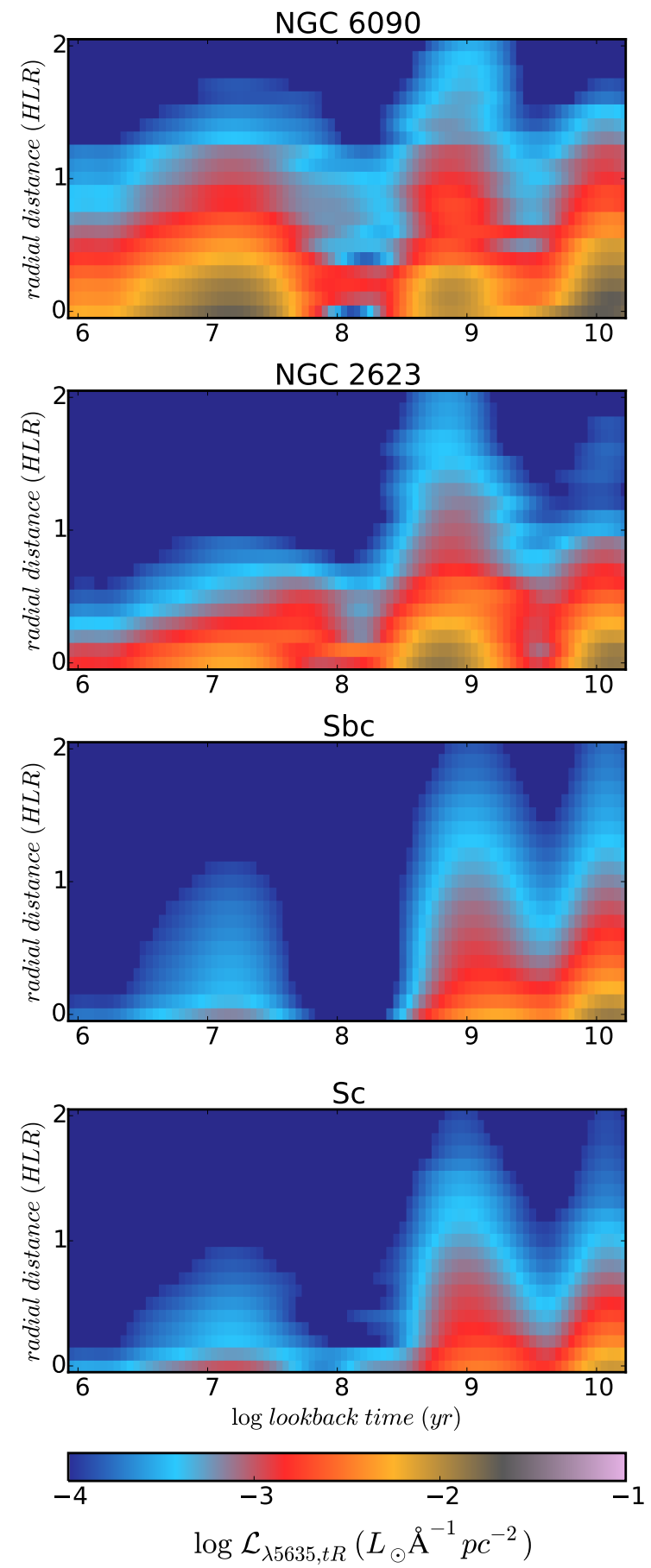
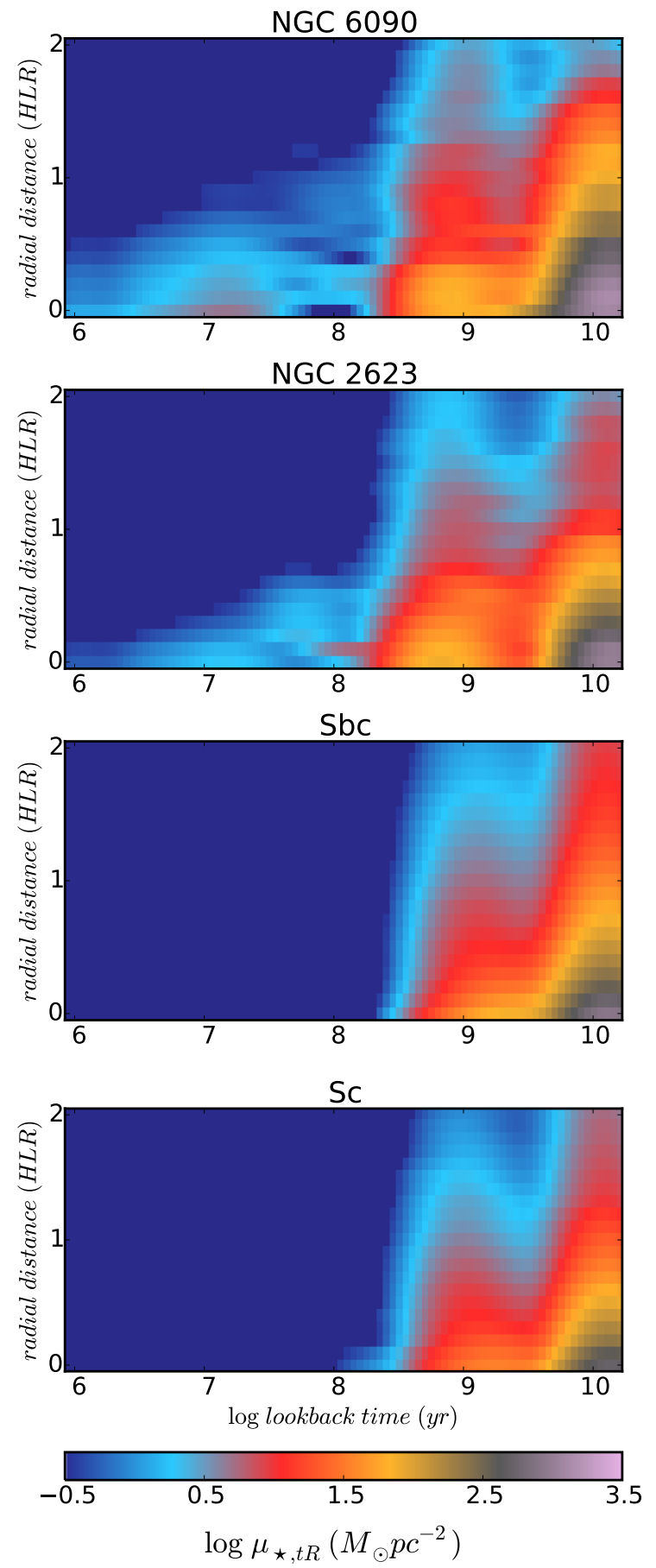

Fig. 2. continued. From top to bottom: $R \times t$ diagrams for NGC 6090, NGC 2623, Sbc, and Sc control spirals.

shown. We note how complex the luminosity and mass densities of LIRG-mergers are in comparison with Sbc/Sc ones. They present prominent spatial and temporal distortions, especially for ages younger than $1 \mathrm{Gyr}$, whose contribution to the luminosity density is significantly higher than in $\mathrm{Sbc/Sc}$ galaxies.

A first way to summarize the SFH is condensing the age distribution in several age ranges. This strategy originated in methods based on fitting equivalent width spectral indices (Bica 1988; Cid Fernandes et al. 2003), but it was also applied to full spectral fits (González Delgado et al. 2004). Here we chose four age scales that typically trace the periods of enhanced star formation in these mergers. They are: 1) $\leq 30 \mathrm{Myr}$, which represents the youngest ionizing stellar populations, and that is comparable to the SFR derived from the $\mathrm{H}_{\alpha}$ (Asari et al. 2007;
González Delgado et al. 2016); 2) 300 Myr which represents young populations emitting in the UV including up to early type A stars; 3) 1 Gyr which are the intermediate age populations dominated by A and F stars; and 4) populations older than 1 Gyr. In order to quantify the dependence of the results with the spatial information, we extract the SFH in three different spatial scales: 1) the central 2 HLR that for each galaxy corresponds approximately to the whole galaxy; 2) the central $0.5 \mathrm{HLR}$, that in early spirals is usually dominated by the bulge component; 3) and between 1 and 1.5 HLR, that usually represents well the average properties of regions located in the disk of spirals. In Table 2 we summarize the contributions in light and mass over the three time scales ( $t \leq 30 \mathrm{Myr}, 300 \mathrm{Myr}$, and $1 \mathrm{Gyr}$ ) and for three spatial regions: from $0-0.5$ HLR, 1-1.5 HLR, and 
C. Cortijo-Ferrero et al.: Star formation history of mergers

Table 2. Light and mass contributions of SSPs over three time scales $(t \leq 30 \mathrm{Myr}, 300 \mathrm{Myr}$, and $1 \mathrm{Gyr})$ and for three spatial regions: from $0-0.5$ HLR (top), 1-1.5 HLR (middle), and 0-2 HLR (bottom).

\begin{tabular}{|c|c|c|c|c|c|c|c|}
\hline \multicolumn{8}{|c|}{$0.0-0.5$ HLR } \\
\hline Light $(\%)$ & Sbc & $\mathrm{Sc}$ & Mice A & Mice B & IC $1623 \mathrm{~W}$ & NGC 6090 & NGC 2623 \\
\hline $\mathrm{SSPs} \leq 30 \mathrm{Myr}$ & $8.0(0.2)$ & $13.3(0.2)$ & $13.5(0.7)$ & $1.4(0.1)$ & $20.5(2.7)$ & $49.2(0.8)$ & $21.7(0.6)$ \\
\hline SSPs $\leq 300 \mathrm{Myr}$ & $8.4(0.2)$ & $17.7(0.1)$ & $17.1(0.7)$ & $1.4(0.1)$ & $57.1(0.9)$ & $52.20(0.02)$ & $29.9(0.3)$ \\
\hline SSPs $\leq 1 \mathrm{Gyr}$ & $19.0(2.0)$ & $32.4(2.9)$ & $27.0(0.9)$ & $4.2(0.4)$ & $70.9(0.9)$ & $67.5(0.9)$ & $64.7(1.1)$ \\
\hline Mass (\%) & Sbc & $\mathrm{Sc}$ & Mice A & Mice B & IC $1623 \mathrm{~W}$ & NGC 6090 & NGC 2623 \\
\hline SSPs $\leq 30 \mathrm{Myr}$ & $0.061(0.004)$ & $0.133(0.006)$ & $0.13(0.02)$ & $0.007(0.002)$ & $0.6(0.1)$ & $0.66(0.02)$ & $0.4(0.1)$ \\
\hline $\mathrm{SSPs} \leq 300 \mathrm{Myr}$ & $0.09(0.01)$ & $0.27(0.01)$ & $0.3(0.1)$ & $0.007(0.002)$ & $4.2(0.3)$ & $0.92(0.01)$ & $1.1(0.1)$ \\
\hline SSPs $\leq 1 \mathrm{Gyr}$ & $2.2(0.5)$ & $4.3(0.9)$ & $2.5(0.4)$ & $0.5(0.1)$ & $7.4(0.3)$ & $6.5(0.4)$ & $10.6(0.7)$ \\
\hline \multicolumn{8}{|c|}{$1.0-1.5 \mathrm{HLR}$} \\
\hline Light $(\%)$ & Sbc & $\mathrm{Sc}$ & Mice A & Mice B & IC $1623 \mathrm{~W}$ & NGC 6090 & NGC 2623 \\
\hline SSPs $\leq 30 \mathrm{Myr}$ & $16.7(0.2)$ & $15.2(0.4)$ & $2.9(0.1)$ & $8.4(0.3)$ & $57.7(0.5)$ & $33.1(1.7)$ & $10.2(0.5)$ \\
\hline SSPs $\leq 300 \mathrm{Myr}$ & $22.7(0.4)$ & $24.2(0.4)$ & $5.1(0.4)$ & $9.5(0.4)$ & $79.7(0.3)$ & $43.9(1.0)$ & $17.8(1.1)$ \\
\hline SSPs $\leq 1 \mathrm{Gyr}$ & $42.5(3.1)$ & $52.8(3.6)$ & $17.8(0.6)$ & $28.1(3.7)$ & $79.7(0.3)$ & $66.2(1.6)$ & $60.9(2.1)$ \\
\hline Mass $(\%)$ & $\mathrm{Sbc}$ & $\mathrm{Sc}$ & Mice A & Mice B & IC $1623 \mathrm{~W}$ & NGC 6090 & NGC 2623 \\
\hline $\mathrm{SSPs} \leq 30 \mathrm{Myr}$ & $0.23(0.01)$ & $0.30(0.02)$ & $0.026(0.001)$ & $0.072(0.004)$ & $1.37(0.01)$ & $0.7(0.1)$ & $0.28(0.02)$ \\
\hline $\mathrm{SSPs} \leq 300 \mathrm{Myr}$ & $0.7(0.1)$ & $1.1(0.1)$ & $0.2(0.1)$ & $0.15(0.02)$ & $3.8(0.1)$ & $1.8(0.1)$ & $1.5(0.3)$ \\
\hline SSPs $\leq 1 \mathrm{Gyr}$ & $7.6(1.3)$ & $13.2(1.8)$ & $2.6(0.2)$ & $3.9(0.8)$ & $3.8(0.1)$ & $11.0(0.9)$ & $23.1(1.7)$ \\
\hline \multicolumn{8}{|c|}{$0.0-2.0$ HLR } \\
\hline Light $(\%)$ & Sbc & $\mathrm{Sc}$ & Mice A & Mice B & IC $1623 \mathrm{~W}$ & NGC 6090 & NGC 2623 \\
\hline SSPs $\leq 30 \mathrm{Myr}$ & $10.5(0.2)$ & $14.0(0.2)$ & $10.2(0.4)$ & $2.9(0.1)$ & $35.5(2.1)$ & $46.8(0.8)$ & $19.3(0.6)$ \\
\hline $\mathrm{SSPs} \leq 300 \mathrm{Myr}$ & $12.2(0.3)$ & $19.4(0.2)$ & $13.5(0.4)$ & $3.2(0.2)$ & $65.6(1.6)$ & $51.1(0.1)$ & $28.3(0.4)$ \\
\hline $\mathrm{SSPs} \leq 1 \mathrm{Gyr}$ & $25.3(2.3)$ & $37.9(3.2)$ & $25.6(1.1)$ & $8.1(1.0)$ & $73.6(0.4)$ & $66.9(1.0)$ & $62.6(1.2)$ \\
\hline Mass (\%) & Sbc & $\mathrm{Sc}$ & Mice A & Mice B & IC $1623 \mathrm{~W}$ & NGC 6090 & NGC 2623 \\
\hline SSPs $\leq 30 \mathrm{Myr}$ & $0.144(0.007)$ & $0.22(0.01)$ & $0.062(0.005)$ & $0.034(0.003)$ & $1.0(0.1)$ & $0.7(0.1)$ & $0.3(0.1)$ \\
\hline SSPs $\leq 300 \mathrm{Myr}$ & $0.36(0.03)$ & $0.6(0.1)$ & $0.2(0.1)$ & $0.08(0.02)$ & $3.7(0.1)$ & $1.2(0.1)$ & $1.3(0.2)$ \\
\hline $\mathrm{SSPs} \leq 1 \mathrm{Gyr}$ & $4.8(0.9)$ & $8.3(1.4)$ & $2.9(0.3)$ & $1.5(0.3)$ & $4.9(0.2)$ & $8.1(0.7)$ & $13.4(0.9)$ \\
\hline
\end{tabular}

0-2 HLR. The uncertainties are shown in brackets, and are the result of propagating the dispersions due to slight age ( \pm 1 SSP) and radial variations $( \pm 0.1 \mathrm{HLR}$ in the central and outer regions, and \pm 0.5 HLR in the integrated $0-2$ HLR range). The uncertainties calculated in this way are quite small. Indeed, the major uncertainties associated to this method come from the models base choice, and the extinction law. For example, using different spectral fits from NGC 2623, we have found: a) when maintaining the same set of SSPs, but changing to Cardelli extinction law (Cardelli et al. 1989), the fraction of light in ages younger than $32 \mathrm{Myr}, x_{<32} \mathrm{Myr}$, presents a maximum increase of up to $+8 \%$ in the $0-0.5$ HLR bin, and a maximum decrease of up to $-6 \%$ for $x_{<300 \mathrm{Myr}}$, but the differences in the mass fractions are significantly smaller. However, the variations in light and mass fractions for the age bin $<1000 \mathrm{Myr}$ are larger. In terms of light, they present a maximum decrease of $-4 \%$ in the 0 0.5 HLR region, and an increase of $+5 \%$ in the 1-1.5 HLR, with mass variations, $m_{<1 \mathrm{Gyr}}$, of $\sim-2 \%$ and $\sim+2 \%$, respectively; b) analogously, when maintaining the Calzetti extinction law but changing the model base to one built from a 2007 update of the Bruzual \& Charlot (2003) models, BC07 models give $x_{<1000 \mathrm{Myr}}$ that are $11 \%$ smaller than with GSD base, but still of $2-3 \%$ in mass fraction. In summary, although these variations are significantly larger than those obtained by the propagation of uncertainties in SSP ages and space intervals, they are still of $\lesssim 2-3 \%$. A brief description of the results based on these quantifications is presented below.

\subsubsection{SFH: Mice $A$ and $B$}

The optical light of the Mice is dominated by the old stellar populations. Populations younger than $1 \mathrm{Gyr}$ contribute, on average, to the luminosity density, with $25.6 \pm 1.1 \% 3$ (Mice A) and $8.1 \pm 1.0 \%$ (Mice B). In the Mice B these populations are more relevant in the $1-1.5$ HLR regions $(28.1 \pm 3.7 \%)$ than in the central 0.5 HLR regions $(4.2 \pm 0.4 \%)$, while in contrast, in Mice A they are less relevant in the $1-1.5$ HLR regions $(17.8 \pm 0.6 \%)$ than in the central $0.5 \mathrm{HLR}(27.0 \pm 0.9 \%)$. Old populations contribute with $74 \%(92 \%)$ to the optical light of the Mice A (B), and dominate the stellar mass density, accounting for more than $97 \%$ of the total mass.

\subsubsection{SFH: IC 1623}

Stellar populations younger than $300 \mathrm{Myr}$ account for more than $65.6 \pm 1.6 \%$ of the luminosity density of this galaxy, being approximately similar in the inner $0.5 \operatorname{HLR}(57.1 \pm 0.9 \%)$, and significant higher $(79.7 \pm 0.3 \%)$ at regions between $1-1.5$ HLR. Populations younger than $30 \mathrm{Myr}$ are also relevant in this galaxy, contributing with $20.5 \pm 2.7 \%$ in the central $0.5 \mathrm{HLR}$, and up to $57.7 \pm 0.5 \%$ in $1-1.5$ HLR regions. Old populations contribute with less than $26 \%$, but these old components account for more than $95 \%$ of the mass.

\footnotetext{
3 Dispersions are only based on those tabulated in Table 2.
} 

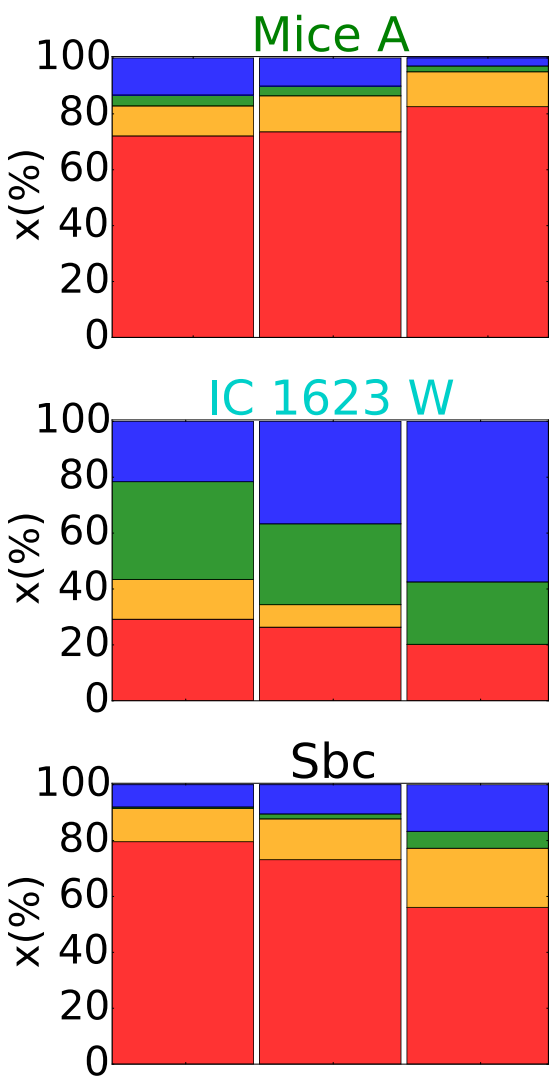
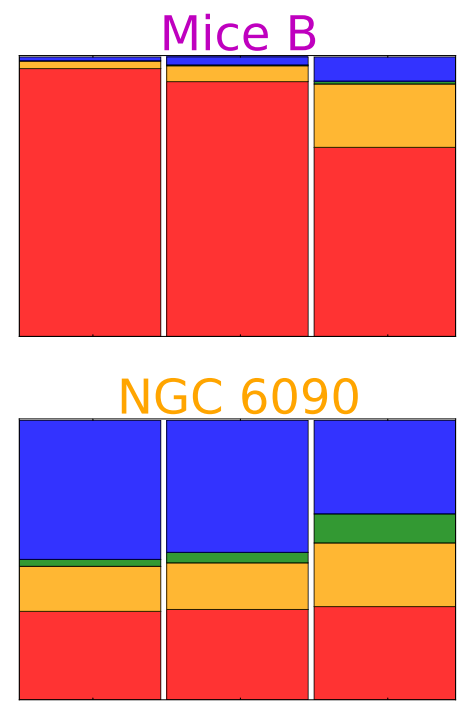

Sc
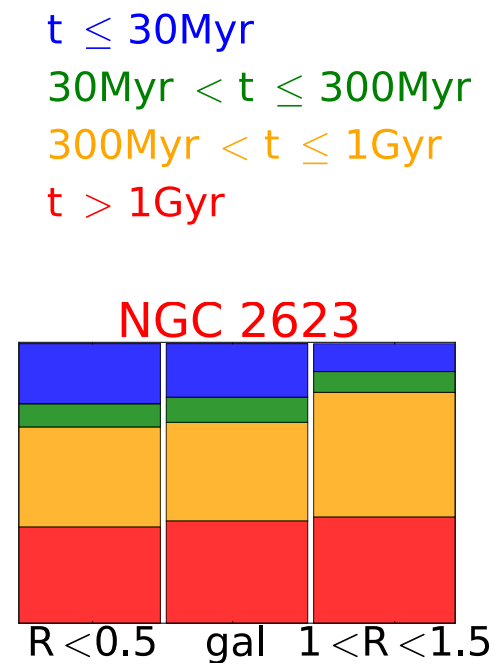

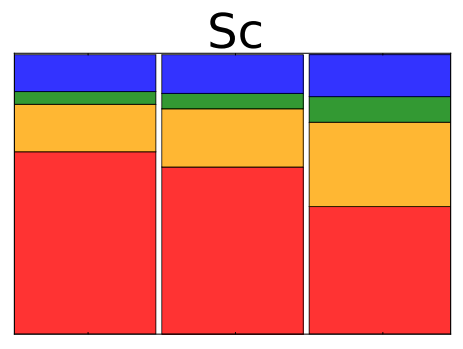

Fig. 3. Average light fractions due to stars in different age and radial ranges. Each panel corresponds to a galaxy merger (first and second rows), and the comparison $\mathrm{Sbc} / \mathrm{Sc}$ galaxies are shown in the third row. The three bar chart histograms in each panel correspond to different galaxy regions: the inner region $R<0.5$ HLR (left bar); the whole galaxy $R<2$ HLR (central bar); and outer regions $1<R<1.5$ HLR (right bar). Colours represent the different age ranges.

\subsubsection{SFH: NGC 6090}

The optical light of this galaxy is dominated by very young stars $(t<30 \mathrm{Myr})$. They contribute to the luminosity density with $46.8 \pm 0.8 \%$, and with similar fractions in the central 0.5 HLR regions $(49.2 \pm 0.8 \%)$, and slightly lower in the $1-1.5$ HLR regions $(33.1 \pm 1.7 \%)$. Stellar populations with ages between $30 \mathrm{Myr}$ and $1 \mathrm{Gyr}$, account in this galaxy by only $\sim 20 \%$, although is higher $(33 \%)$ in outer regions. Old populations contribute with $\sim 34 \%$ to the optical light, and dominate the stellar mass density, as in IC 1623 by $91 \%$ of the total mass.

\subsubsection{SFH: NGC 2623}

Young $(t<30 \mathrm{Myr}$ ) and intermediate (30 Myr $<t<1 \mathrm{Gyr}$ ) stars are relevant in this galaxy, they contribute with $\sim 19.3 \pm 0.6 \%$ and $43.3 \pm 1.3 \%$ each one to the total light density, and with similar fractions, $\sim 21.7 \pm 0.6 \%$ and $\sim 43.0 \pm 1.3 \%$ for the young and intermediate ages, respectively, in the central 0.5 HLR, and in $1-1.5$ HLR these fractions are $\sim 10.2 \pm 0.5 \%$ and $\sim 50.7 \pm$ $1.8 \%$. Populations older than $1 \mathrm{Gyr}$ contribute $\sim 37 \%$ of the light density, and with $86 \%$ of the mass density, on average in 0-2 HLR region, being this contribution similar in the central region $(89 \%)$, and slightly lower in the disk $(77 \%)$.

\subsection{SFH: light fraction}

To compare the similarities and differences between the SFH of these galaxies, Fig. 3 presents an alternative way of visualizing the results by discretizing the light fraction $(x)$ in a few relevant
$R$ and $t$ ranges. Each panel shows three rectangular bar charts, each corresponding to a spatial region: $R<0.5$ HLR (left), $0<$ $R<2$ HLR (middle), and $1<R<1.5$ HLR (right). The middle charts are meant to represent the galaxy as a whole. The age information is compressed into four representative ranges: $t \leq$ $30 \mathrm{Myr}, 30 \mathrm{Myr}<t \leq 300 \mathrm{Myr}, 300 \mathrm{Myr}<t \leq 1 \mathrm{Gyr}$, and $t>1$ Gyr, tracing the main epochs where merger-induced star formation is triggered.

The most important difference that emerges between the LIRG-mergers (IC 1623, NGC 6090, and NGC 2623) and disk galaxies is that in the former there is a significantly larger light fraction in $\lesssim 1$ Gyr components than in Sbc and Sc galaxies. This is clearly evident in the inner $(<0.5$ HLR) for the 3 LIRGmergers and also in the outer regions (1-1.5 HLR) for the premerger LIRGs IC 1623 and NGC 6090. This is expected because of their FIR luminosity, these galaxies had to experience a burst of star formation in the last $1 \mathrm{Gyr}$, and stellar populations of ages younger than 1 Gyr dominate the light at optical wavelengths, as we have seen in the descriptions above.

The SFH of the Mice, however, are more similar to spirals than to the LIRG-mergers, in the sense that most of the optical light is dominated by stellar populations of $1 \mathrm{Gyr}$ and older. In particular, the outer regions of Mice A and Mice B seem to have suppressed the star formation in the last $<1$ Gyr in comparison with the control spirals.

Using Table 2, we have quantified this in more detail:

- Inner 0.5 HLR: we found that the contributions to light of the youngest SSPs (<30 Myr) in IC $1623 \mathrm{~W}$, NGC 6090, and NGC 2623 are respectively a factor of three (two), six (four), 
and three (two) times higher than in Sbc (Sc) galaxies. In contrast, in Mice A are similar to Sbc (Sc) galaxies, by two (one), and significantly suppressed in Mice B, by factors of six (13) lower than in Sbc (Sc) galaxies. Also, when integrating over the last $300 \mathrm{Myr}$, the light fractions we measure in IC $1623 \mathrm{~W}$, NGC 6090, and NGC 2623 are a factor of seven (three), six (three), and four (two) higher than the light fraction in $\mathrm{Sbc}(\mathrm{Sc})$ galaxies. In Mice $\mathrm{A}$ is similar to $\mathrm{Sbc}(\mathrm{Sc})$ galaxies, and suppressed in Mice B, as in the youngest time scale. Lastly, the central star formation in the last $1 \mathrm{Gyr}$ is also enhanced in the three LIRGs with respect to the control spirals by a factor of four-to-three (two), in terms of light, with respect to $\mathrm{Sbc}(\mathrm{Sc})$. This is approximately a factor of approximately two less than what we measured for the shorter $300 \mathrm{Myr}$ time scale. This indicates that the star formation has not been continuous in the last $1 \mathrm{Gyr}$ but rather in bursts. In Mice A, the star formation in the $1 \mathrm{Gyr}$ time scale is similar to the control spirals, and suppressed in Mice B by the same amount as in the other time scales.

- 1-1.5 HLR regions: in the outer-regions of the two earlystage mergers IC $1623 \mathrm{~W}$ and NGC 6090, the star formation in the last $30 \mathrm{Myr}$ is, respectively, a factor of approximately four and two higher in light fraction than the average of the control Sbc and Sc. Otherwise, in the merger NGC 2623 the contribution is comparable or slightly lower $0.6(0.7)$ than in $\mathrm{Sbc}(\mathrm{Sc})$ galaxies, suggesting that the star formation in the last 30 Myr has been significantly enhanced in the central region of NGC 2623 but not in its "disk". Again, in the outerregions of the early-stage merger LIRGs, the star formation in the last $300 \mathrm{Myr}$ is a factor of three (for IC $1623 \mathrm{~W}$ ) and two (for NGC 6090) higher than in the control spirals. The contribution to light of the SSPs < $300 \mathrm{Myr}$ in NGC 2623 is comparable to the one in the control spirals. In terms of light, the star formation in the three LIRGs in the last $1 \mathrm{Gyr}$ is not enhanced (or very slightly) with respect to the control spirals. In the disk of both Mice galaxies, the star formation in the three time scales is suppressed with respect to $\mathrm{Sbc}(\mathrm{Sc})$ galaxies by $0.3-0.4$ in Mice A, and 0.3-0.5 in Mice B.

- Central 2.0 HLR, global average: the global average results are intermediate between the ones described above.

In conclusion, in the two early-stage merger LIRGs the star formation in the last $30 \mathrm{Myr}$, also in the last $300 \mathrm{Myr}$, is enhanced with respect to the control spirals both in the central regions and in the disks. In the merger NGC 2623, the young star formation in the last $30 \mathrm{Myr}$ and $300 \mathrm{Myr}$ is significantly enhanced in the central region, while in the outer parts it is comparable to the Sbc-Sc spirals. In contrast, in Mice A and B there is no evidence of enhanced SF with respect to isolated spirals; on the contrary, it is suppressed in the disk of Mice A and both in the disk and centre of Mice B.

\subsection{SFH: mass assembly}

Figure 4 presents the cumulative functions of the SFH, showing the mass growth curve as a function of lookback time. This is obtained by adding all the mass formed in the system up to a given lookback time and dividing by the present day stellar mass. This cumulative mass function is derived by adding the contribution of regions located at: (i) the central 0.5 HLR, (ii) between 1 and 1.5 HLR, and (iii) adding almost the whole galaxy (between 0 and 2 HLR). The uncertainties are shaded in light colours. They are calculated as the dispersion in the profiles due to \pm 0.1 HLR variations in the radial distance in the $0-0.5$ HLR and 1-1.5 HLR regions, and \pm 0.5 HLR for the global 0-2 HLR region.
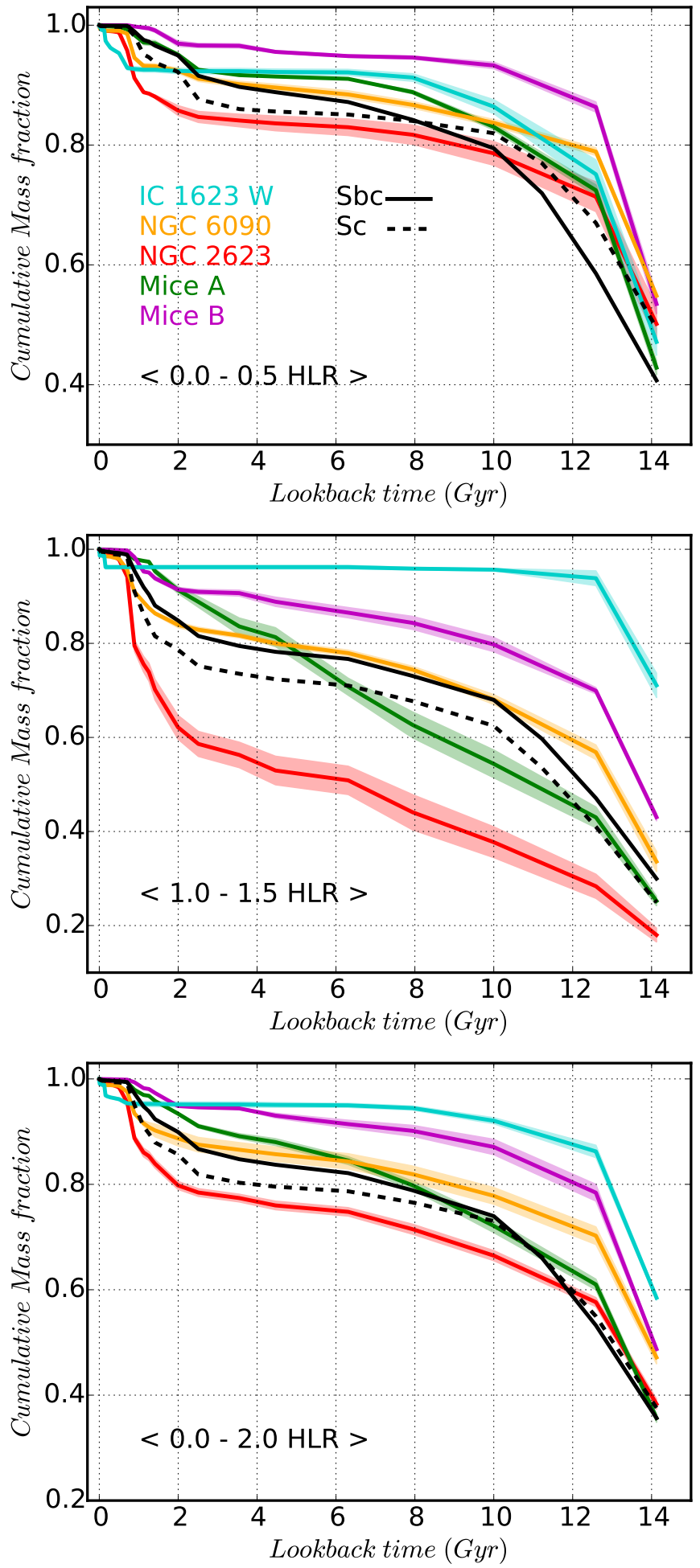

Fig. 4. Cumulative mass growth for the inner 0.5 HLR (upper panel), between 1 and 1.5 HLR (middle panel), and the central 2 HLR (bottom panel). The uncertainties are shaded in light colours, calculated as the dispersion in the profiles due to \pm 0.1 HLR variations in the radial distance in the $0-0.5$ HLR and 1-1.5 HLR regions, and \pm 0.5 HLR for the global 0-2 HLR region. The merger galaxies are colour coded, and they are compared with the results obtained for Sbc (black line) and Sc (dashed-black line) galaxies.

As expected, the young stellar population ( $<1 \mathrm{Gyr})$ that dominated the optical light contributes little to the mass; and population older than 2 Gyr are the main contributors. This is also 
true for the Sbc and Sc galaxies, where the populations older than 10 Gyr contribute with $\sim 73 \%$ of the total mass. These old stellar populations also contribute significantly to the mass in these mergers, with a fraction than range between $\sim 66 \%$ for the NGC 2623 , to $\sim 92 \%$ for IC 1623 . However, in terms of mass assembly the SFH of mergers are diverse, and present interesting differences and similarities to the SFH of late-type spiral galaxies. The most relevant are: a) the mass growth in these mergers, as also in Sbc-Sc galaxies, was very fast, although the growth rate is different for each galaxy. Globally, mergers have grown their mass faster or at similar rate than Sbc and Sc galaxies, except in NGC 2623, where outer regions have grown more slowly; b) except in IC 1623, the mass is assembled faster in the inner part than in the outskirts (compare middle and upper panels Fig. 4). These galaxy mergers have grown most of their mass inside-out, as we have found in most of the CALIFA galaxies (Pérez et al. 2013; García-Benito et al. 2017).

To quantify the rate at which these galaxies have grown their mass, we calculate the epoch $\left(t_{80}\right)$ at which roughly $\sim 80 \%$ of the present stellar mass had been assembled.

- Inner 0.5 HLR: the central regions of these mergers formed at similar epoch than in Sbc $(\mathrm{Sc})$ galaxies. In particular, $t_{80}$ was 12.9 Gyr ago in Mice B, 12.0 Gyr ago in NGC 6090, $11.4 \mathrm{Gyr}$ ago in IC $1623 \mathrm{~W}, 10.7 \mathrm{Gyr}$ ago in Mice A, 9.7 Gyr (10.5 Gyr) ago in Sbc (Sc) galaxies, and 9 Gyr ago in NGC 2623. We find that the mass contribution of SSPs $<$ $1 \mathrm{Gyr}$ in the central regions of the two early-stage mergers IC $1623 \mathrm{~W}$ and NGC 6090 is a factor of approximately two higher than in Sbc-Sc spirals, while it is a factor of approximately three higher for the merger NGC 2623. In contrast, in Mice A it is comparable to Sbc-Sc galaxies, and suppressed by a factor eight in Mice $\mathrm{B}$.

- 1-1.5 HLR: the outer regions of all the early-stage mergers (IC 1623 W, NGC 6090, and Mice) formed earlier than $\mathrm{Sbc}(\mathrm{Sc})$ galaxies, and these earlier than the merger LIRG NGC 2623. Quantitatively, $t_{80}$ was 13.5 Gyr ago in IC 1623 W, 9.8 Gyr ago in Mice B, 4.7 Gyr ago in Mice A, 4.4 Gyr ago in NGC 6090, 3.3 Gyr ago (1.7 Gyr ago) in Sbc (Sc), and 0.9 Gyr ago in NGC 2623. Also, beyond 1 HLR, NGC 2623 formed twice as much mass fraction in the last 1 Gyr than Sbc and Sc galaxies. This increment is similar, but slightly lower, to the one found in the central part (approximately three). In the early-stage merger LIRG NGC 6090 the relative mass formed in the last $1 \mathrm{Gyr}$ is comparable to Sbc-Sc spirals and in IC $1623 \mathrm{~W}$ it is a factor of three less, as if the star formation would have been inhibited in this system. The same happens in Mice A and Mice B, that have formed factors of five and two, respectively, less mass than the control spirals.

- Central 2.0 HLR: the global average results are something similar, and intermediate, between the ones already derived in the central and the outer regions. Quantitatively, $t_{80}$ was there 12.9 Gyr ago in IC $1623 \mathrm{~W}, 12.1 \mathrm{Gyr}$ ago in the Mice B, 8.9 Gyr ago in NGC 6090, 7.8 Gyr ago in the Mice A, 7.3 Gyr ago (3.9 Gyr ago) in $\mathrm{Sbc}(\mathrm{Sc})$, and 1.9 Gyr ago in NGC 2623. Again, from the global averages, only NGC 2623 has formed in the last 1 Gyr a factor of two more mass fraction than the control spirals, while for the early-stage merger LIRGs it is comparable or even lower.

In summary, we conclude that most of the mass was assembled in the main body of these early-stage merger LIRGs very early on, similarly to the same epoch of early type spirals and massive galaxies (González Delgado et al. 2017); but in NGC 2623 it has happened later than in the early-stage mergers and Sbc-Sc. In terms of the mass formed in the last $1 \mathrm{Gyr}$, in the early-stage merger LIRGs it is enhanced by a factor of two in the central regions compared to $\mathrm{Sbc/Sc}$ galaxies, but comparable to them in the disks (or even lower as in the external regions of IC $1623 \mathrm{~W}$ ). In contrast, the merger NGC 2623 has formed more mass fraction than the control spirals in this period, by a factor of three in the central regions and two in the outer regions. Mice A (B) formed, on average, a factor of two (four) less mass fraction in the last 1 Gyr than Sbc-Sc galaxies.

\section{Spatially resolved recent star formation rates}

This section presents the radial structure of the intensity of the star formation rate $\left(\Sigma_{\mathrm{SFR}}\right)$ and the local specific star formation rate of the merger galaxies and the comparison with the control spiral galaxies Sbc-Sc. These two quantities are very suitable to obtain spatially resolved information about the rate at which stars form in a galaxy in recent epochs, and are independent of the galaxy size or stellar mass. These two properties have been well characterized for the CALIFA sample as a function of Hubble type by González Delgado et al. (2016, 2017), and constitute a very rich and a highly valuable source of comparison between mergers and non-interacting spiral galaxies. First, we need to briefly explain the choices of the recent star formation time scales.

\subsection{Choices for the star formation time scale}

From the SFH we can calculate the SFR as the ratio of the mass formed during a given time interval to the duration of this time interval. This represents a time-averaged star formation during each time step. To compute the recent star formation rate, we need to specify what we mean by "recent past" by defining $t_{\mathrm{SF}}$ as the age of the oldest stars to be included in the computation of our recent SFR. The mean rate of star formation is calculated in each $x y$ position in the galaxy by summing over all the populations younger than $t_{\mathrm{SF}}$ and dividing by $t_{\mathrm{SF}}$.

Although the choice of $t_{\mathrm{SF}}$ is arbitrary, we have already found that $t_{\mathrm{SF}}=30 \mathrm{Myr}$ is a good choice because it shows the best correlation between the stellar and $\mathrm{H} \alpha$ based estimates of the SFR (González Delgado et al. 2016; see also Asari et al. 2007, that for their study of star forming galaxies with SDSS found $t_{\mathrm{SF}}=25 \mathrm{Myr}$ ). This choice also coincides with the life time of $\mathrm{O}$ and early type $\mathrm{B}$ stars, which are the major contributors to the ionized gas in galaxies.

Considering the relevance of the intermediate age stellar populations in these mergers, we also need to define two complementary star formation time scales: $t_{\mathrm{SF}}=300 \mathrm{Myr}$, which represents young populations emitting in the UV including up to early type A stars; and $t_{\mathrm{SF}}=1 \mathrm{Gyr}$, which are the intermediate age populations dominated by $\mathrm{A}$ and $\mathrm{F}$ stars. These choices are similar to those proposed by Pereira-Santaella et al. (2015) that found that the UV and FIR diagnostics trace well the SFR on scales of $\sim 100$ Myr and 1 Gyr in a sample of local LIRGs. Thus, in the next sections we will obtain $\Sigma_{\mathrm{SFR}}$ and the local sSFR by using these three time scales for the star formation: $t_{\mathrm{SF}}=30 \mathrm{Myr}$, 300 Myr, and 1 Gyr.

\subsection{The intensity of the star formation rate, $\Sigma_{\mathrm{SFR}}$}

The intensity of star formation rate is defined as the rate of star formation per area; that is the star formation rate surface density. 

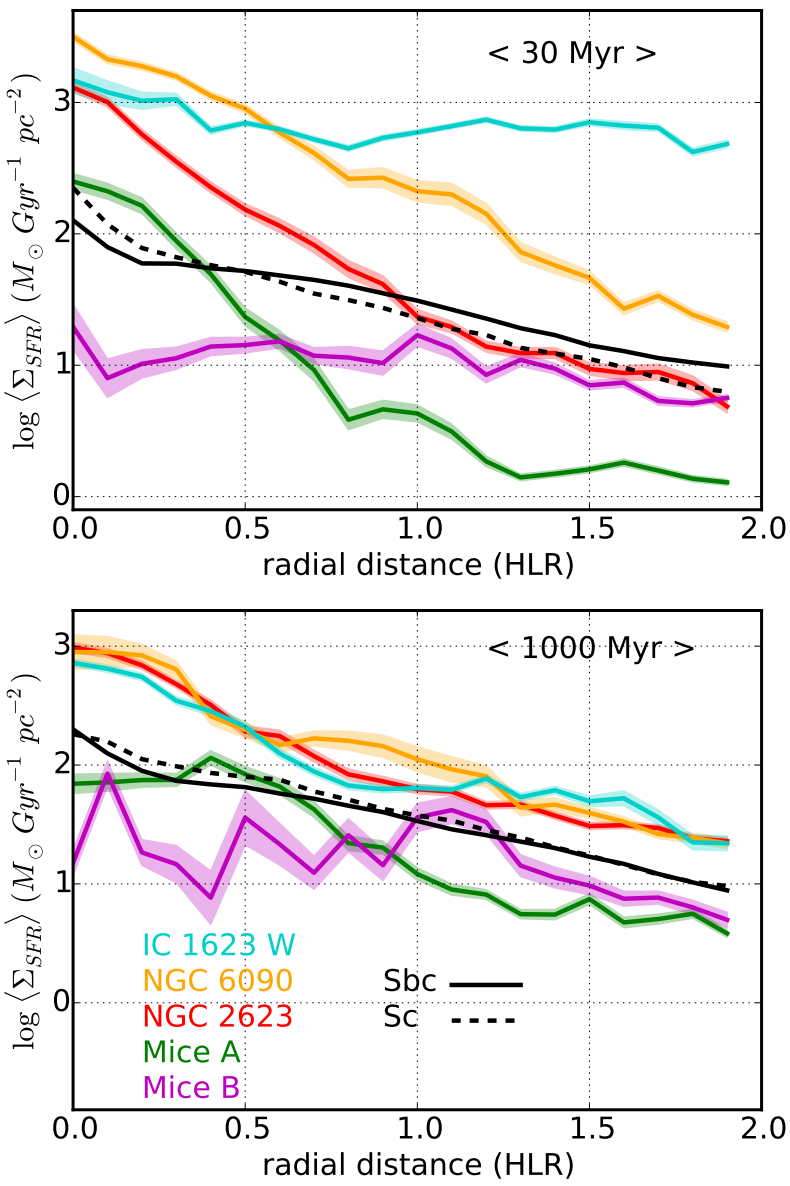

Fig. 5. Radial profiles of the intensity of the star formation rate $\left(\Sigma_{\mathrm{SFR}}\right)$ obtained for two different time scales: the last $30 \mathrm{Myr}$, and $1.0 \mathrm{Gyr}$. The uncertainties, calculated as the standard error of the mean in each \pm 0.1 HLR bin, are shaded in light colours.

We compute $\Sigma_{\mathrm{SFR}}\left(t_{\mathrm{SF}}\right)$ from the $\mu_{\star, t R} 2 \mathrm{D}$ maps by adding the contribution of components with $t \leq t_{\mathrm{SF}}$, and dividing by the $t_{\mathrm{SF}}$ time scale.

Previously, in the analysis of spiral galaxies, we found that $\Sigma_{\mathrm{SFR}}$ at the present time shows declining radial profiles that exhibit very little differences between spirals; at a given radial distance, there is a small dispersion in $\Sigma_{\mathrm{SFR}}$ that can be understood because galaxies in the main sequence of star formation (MSSF) have nearly constant SFI. A comparison of the $\Sigma_{\mathrm{SFR}}$ radial profiles of the mergers obtained for different $t_{\mathrm{SF}}$ and those of $\mathrm{Sbc}-\mathrm{Sc}$ galaxies can tell us if there is an enhanced SFR in these mergers, where does it happen, and over which time scale.

This comparison for $t_{\mathrm{SF}}=30 \mathrm{Myr}$ and $1 \mathrm{Gyr}$ is shown in Fig. 5. The uncertainties are shaded in light colours. They represent the standard error of the mean, calculated as the standard deviation divided by the square root of the number of points in each distance bin, with 0.2 HLR of width. As it is normalized by the square root of the number of points or spaxels in each HLR bin, the differences in the uncertainties between the inner and outer regions are visually not so strong, as the larger the radius, the larger is $N$. In terms of dispersion (=standard deviation) we find that with respect to the centre, the dispersion is, on average, a factor of approximately two larger at $0.5 \mathrm{HLR}$, approximately three larger at $1 \mathrm{HLR}$, and approximately five at 2 HLR.

For the time scale $<30 \mathrm{Myr}$, the $\Sigma_{\mathrm{SFR}}$ in LIRG-merger galaxies is enhanced with respect to spirals. On the contrary, the Mice show a significant depression of the star formation with respect to the LIRG-mergers. Although at the inner 0.5 HLR of Mice A,
$\Sigma_{\mathrm{SFR}}$ is similar to Sc galaxies, in the outer regions, and in Mice B, $\Sigma_{\mathrm{SFR}}$ is significantly below the control spirals. In the time scale $t_{\mathrm{SF}}=1 \mathrm{Gyr}$, the LIRG-mergers also show some enhancement of the SFR with respect to Sbc-Sc galaxies, but the radial structure of $\Sigma_{\mathrm{SFR}}$ of the three mergers is more similar and also similar to the declining profile in spirals. $\Sigma_{\mathrm{SFR}}$ in Mice A also shows a declining profile, being in the central $\sim 0.5$ HLR similar to the control spirals. In Mice B, $\Sigma_{\mathrm{SFR}}$ is more variable, and significantly depressed with respect to Sbc galaxies in the central 1 HLR.

Quantifying the results in more detail:

- $t_{\mathrm{SF}}=30 \mathrm{Myr}$ : Taking as the reference the inner 0.5 HLR, we find that the $\Sigma_{\mathrm{SFR}}\left(t_{\mathrm{SF}}=30 \mathrm{Myr}\right)$ of IC $1623 \mathrm{~W}$, NGC 6090, and NGC 2623 are enhanced by $1.15 \mathrm{dex}$ (1.05 dex), $1.38 \mathrm{dex}$ (1.28 dex), and $0.83 \mathrm{dex}(0.72 \mathrm{dex})$, respectively, with respect to the average of $\mathrm{Sbc}(\mathrm{Sc})$ galaxies, while it is much more similar for Mice A, 0.16 dex (0.06 dex), and significantly depressed in Mice B, by -0.74 dex ( -0.84 dex). Analogously, taking now as reference the outer parts from 1-1.5 HLR, we find differences between our mergers and Sbc (Sc) galaxies of $1.50 \mathrm{dex}(1.63 \mathrm{dex})$ for IC $1623 \mathrm{~W}$, 0.69 dex $(0.82 \mathrm{dex})$ for NGC $6090,-0.16 \mathrm{dex}(-0.03 \mathrm{dex})$ for NGC $2623,-1.00$ dex $(-0.87 \mathrm{dex})$ for Mice A, and -0.30 dex $(-0.17$ dex $)$ for Mice B. These results indicate that in the two early-stage merger LIRGs the star formation in the last $30 \mathrm{Myr}$ is enhanced with respect to the control spirals both in the inner and outer regions, while for the merger NGC $2623, \Sigma_{\mathrm{SFR}}\left(t_{\mathrm{SF}}=30 \mathrm{Myr}\right)$ is significantly enhanced in the central region, but at the outer parts it is similar or even slightly decreased in comparison to the control spirals. On the contrary, the only region of the Mice with $\Sigma_{\mathrm{SFR}}\left(t_{\mathrm{SF}}=30 \mathrm{Myr}\right)$ similar to $\mathrm{Sbc}-\mathrm{Sc}$ galaxies is the centre of Mice A; in its disk and the whole Mice B is significantly decreased. With respect to the gradients, in the inner $1 \mathrm{HLR}^{4}$ we found that for $\Sigma_{\mathrm{SFR}}\left(t_{\mathrm{SF}}=30 \mathrm{Myr}\right)$ the gradient is negative for the mergers and control spirals, with values of $-0.40 \mathrm{dex} / \mathrm{HLR}$ in IC $1623 \mathrm{~W},-1.18 \mathrm{dex} / \mathrm{HLR}$ in NGC 6090, $-1.74 \mathrm{dex} / \mathrm{HLR}$ in NGC 2623, $-1.77 \mathrm{dex} / \mathrm{HLR}$ in Mice A, $-0.06 \mathrm{dex} / \mathrm{HLR}$ in Mice $\mathrm{B},-0.61 \mathrm{dex} / \mathrm{HLR}$ in Sbc, and -0.99 dex/HLR in Sc galaxies. The main differences are in the slope, while in NGC 6090 it is only a bit steeper than in Sc galaxies, in NGC 2623 and Mice A it is significantly steeper, and in IC $1623 \mathrm{~W}$ and Mice B the gradient is even flatter than in Sbc galaxies. A negative gradient indicates a central triggering of the star formation in comparison with that at 1 HLR. Although not shown, the results for the longer young-intermediate time scale $\left(t_{\mathrm{SF}} \lesssim 300 \mathrm{Myr}\right)$ are qualitatively consistent with these for $t_{\mathrm{SF}}=30 \mathrm{Myr}$.

- $t_{\mathrm{SF}}=1 \mathrm{Gyr}$ : We found that in the inner 0.5 HLR the difference in $\Sigma_{\mathrm{SFR}}\left(t_{\mathrm{SF}}=1 \mathrm{Gyr}\right)$ between our mergers and Sbc (Sc) galaxies is $0.64 \mathrm{dex}(0.57 \mathrm{dex})$ in IC $1623 \mathrm{~W}, 0.74 \mathrm{dex}$ $(0.67 \mathrm{dex})$ in NGC 6090, $0.73 \mathrm{dex}(0.65 \mathrm{dex})$ in NGC 2623, -0.07 dex $(-0.15$ dex $)$ in Mice A, and -0.65 dex $(-0.73$ dex $)$ in Mice B. Analogously, taking now as reference the outer parts from $1-1.5 \mathrm{HLR}, \Sigma_{\mathrm{SFR}}\left(t_{\mathrm{SF}}=1 \mathrm{Gyr}\right)$ of IC $1623 \mathrm{~W}$, NGC 6090, NGC 2623, Mice A, and Mice B, differ from the $\mathrm{Sbc}(\mathrm{Sc})$ galaxies by $0.40 \mathrm{dex}(0.37 \mathrm{dex}), 0.42 \mathrm{dex}$ (0.39 dex), 0.28 dex (0.24 dex), -0.50 dex $(-0.53$ dex $)$, and -0.07 dex $(-0.10$ dex $)$, respectively. Thus, in the central $0.5 \mathrm{HLR}$ star formation in the last $1 \mathrm{Gyr}$ is enhanced in IC $1623 \mathrm{~W}$, NGC 6090, and NGC 2623 with respect to control spirals $(\leq 5$, significantly less than for the young

$4 \nabla_{\text {in }} \log \Sigma_{\mathrm{SFR}}=\log \Sigma_{\mathrm{SFR}}(1 \mathrm{HLR})-\log \Sigma_{\mathrm{SFR}}(0)$, as in González Delgado et al. (2015). 
$30 \mathrm{Myr}$ time scale), and in the outer parts (1-1.5 HLR) of the LIRGs $\Sigma_{\mathrm{SFR}}\left(t_{\mathrm{SF}}=1 \mathrm{Gyr}\right)$ is only slightly enhanced with respect to the control spirals. At this time scale, the Mice show a decreased star formation, in comparison with $\mathrm{Sbc}-\mathrm{Sc}$ galaxies, except maybe in the centre of Mice A, where it is similar to Sbc galaxies. With respect to the gradients, $\Sigma_{\mathrm{SFR}}\left(t_{\mathrm{SF}}=1 \mathrm{Gyr}\right)$ gradient in the inner $1 \mathrm{HLR}$ is $-1.05 \mathrm{dex} / \mathrm{HLR}$ for IC $1623 \mathrm{~W},-0.90 \mathrm{dex} / \mathrm{HLR}$ for NGC 6090, -1.19 dex/HLR for NGC 2623, -0.76 dex/HLR for Mice A, $0.38 \mathrm{dex} / \mathrm{HLR}$ for Mice B, $-0.77 \mathrm{dex} / \mathrm{HLR}$ for Sbc galaxies, and $-0.68 \mathrm{dex} / \mathrm{HLR}$ for Sc. Except for Mice B, that shows a flat gradient, the rest of them have a negative gradient, with the one in NGC 6090 and Mice A being similar to Sbc galaxies, and in IC 1623 W and NGC 2623 steeper than for the control spirals.

\section{3. $S S F R$}

As in González Delgado et al. (2016), we obtain the local specific star formation rate as the ratio between the intensity of the recent star formation rate and the stellar mass surface density, $s S F R(R)=\Sigma_{\mathrm{SFR}}(R) / \mu_{\star}(R)$. It measures the relative rate of ongoing star formation with respect to the past in each position in a galaxy. This local definition is equivalent to the global sSFR defined for a galaxy as the ratio between the total SFR and the galaxy stellar mass.

For $t_{\mathrm{SF}}=30 \mathrm{Myr}$ we have found for spiral galaxies that $\operatorname{sSFR}(R)$ increases outwards as a function of the radial distance, growing faster in the inner 1 HLR than outwards, probably signaling the bulge-disk transition, and the effect of the quenching that progresses inside-out of galaxies (Tacchella et al. 2015; González Delgado et al. 2016).

The local sSFR allows to compare the relative star formation between different regions, as it is normalized by the amount of stellar mass. Figure 6 shows the radial profiles of sSFR of the mergers and control spirals; the comparisons are done using different star forming time scales as defined above. The first result to note is that in the time scale of $30 \mathrm{Myr}$, the radial profiles $\Sigma_{\mathrm{SFR}}(R) / \mu_{\star}(R)$ of LIRG-mergers are flatter than in spirals, and above or equal to $0.1 \mathrm{Gyr}^{-1}$. This is an interesting result because $s S F R=0.1 \mathrm{Gyr}^{-1}$ is a threshold to separate star-forming galaxies from quiescent systems (Peng et al. 2010). Thus, the disk regions $(R>1 \mathrm{HLR})$ of our spiral control sample and the LIRGmergers of the sample are above this threshold. But Mice are significantly below, indicating that at the present epoch this system is forming stars with an efficiency that is significantly below to what they did in the past.

Specifying the results in more detail:

- $t_{\mathrm{SF}}=30 \mathrm{Myr}$ : The $\operatorname{sSFR}\left(t_{\mathrm{SF}}=30 \mathrm{Myr}\right)$ in the central 0.5 HLR of IC $1623 \mathrm{~W}$, NGC 6090, and NGC 2623 is respectively $0.93 \mathrm{dex}(0.67 \mathrm{dex}), 0.94 \mathrm{dex}(0.68 \mathrm{dex})$, and $0.65 \mathrm{dex}$ (0.38 dex) higher than in Sbc ( $\mathrm{Sc}$ ) galaxies. In Mice A is similar to Sbc (Sc) galaxies, by 0.15 dex $(-0.12 \mathrm{dex})$, and in Mice B significantly decreased, -1.03 dex $(-1.30$ dex $)$. In the outer parts $(1-1.5 \mathrm{HLR})$, we find that $\operatorname{sSFR}\left(t_{\mathrm{SF}}=\right.$ $30 \mathrm{Myr}$ ) is enhanced with respect $\mathrm{Sbc}(\mathrm{Sc})$ galaxies in the early-stage merger LIRGs IC $1623 \mathrm{~W}, 0.69$ dex (0.68 dex), and NGC 6090, $0.36 \mathrm{dex}(0.33 \mathrm{dex})$, similar to them in NGC 2623, -0.04 dex ( -0.07 dex $)$, and decreased in Mice A and Mice B by $-1.04 \operatorname{dex}(-1.07 \mathrm{dex})$ and $-0.57 \mathrm{dex}$ $(-0.60$ dex $)$, respectively. With respect to the gradient in the inner $1 \mathrm{HLR}$, it is almost flat in NGC 6090 (0.00 dex/HLR), and NGC $2623(-0.23$ dex/HLR) in comparison to the
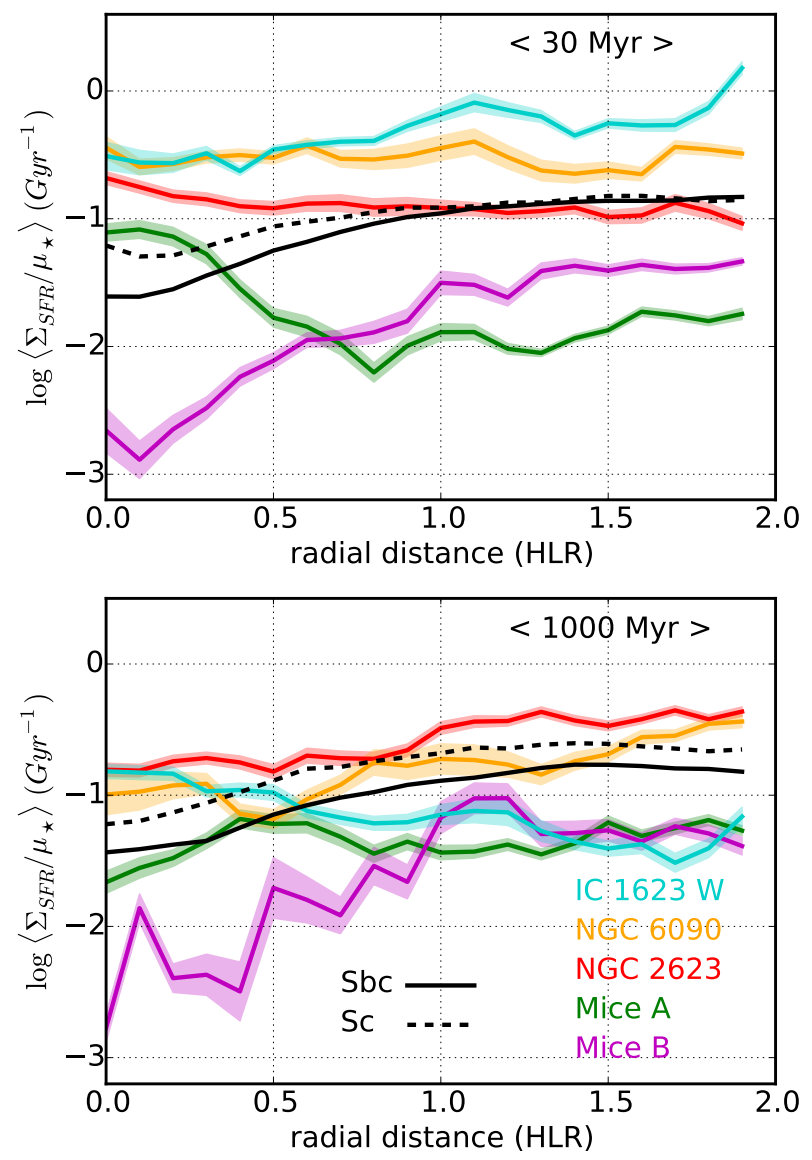

Fig. 6. Radial profiles of the specific star formation rate $\left(\Sigma_{\mathrm{SFR}} / \mu_{\star}\right)$ obtained for two different time scales: the last $30 \mathrm{Myr}$, and $1.0 \mathrm{Gyr}$. The uncertainties, calculated as the standard error of the mean, are shaded in light colours.

clearly positive gradient in $\mathrm{Sbc}(0.65 \mathrm{dex} / \mathrm{HLR})$ and $\mathrm{Sc}(0.30 \mathrm{dex} / \mathrm{HLR})$ galaxies. The positive gradient in the control spirals indicates a central shut down of the star formation in comparison with that at $1 \mathrm{HLR}$, and that the quenching is progressing inside-out (González Delgado et al. 2016). However, the flattening of the local $\operatorname{sSFR}(R)$ in the mergers indicates that these galaxies are forming stars more actively than in the past. In IC $1623 \mathrm{~W}(0.33 \mathrm{dex} / \mathrm{HLR})$ the gradient is positive and more similar to $\mathrm{Sc}$ galaxies, pointing to less recent star formation in the centre than at 1 HLR. By contrast, in Mice A (-0.78 dex/HLR) the gradient is negative but steeper than in the LIRGs, indicating that the increase of the star formation in the last $30 \mathrm{Myr}$ is more important in the nucleus that in the disk. In Mice B (1.16 dex/HLR) the gradient is positive, but steeper than in the control spirals.

Again, when considering the young-intermediate time scale $\left(t_{\mathrm{SF}} \lesssim 300 \mathrm{Myr}\right)$ the results are approximately consistent with the $t_{\mathrm{SF}} \lesssim 30 \mathrm{Myr}$ ones. The star formation is enhanced in the central regions of IC $1623 \mathrm{~W}$ (by a factor 29), NGC 6090 (by a factor of six), and NGC 2623 (by a factor of seven) in comparison with the control spirals. In the outer parts, only IC $1623 \mathrm{~W}$ presents a significant enhancement (by a factor four) of the star formation with respect to control spirals. On the contrary, in the outer parts of NGC 6090, and NGC 2623, the star formation is not so significantly increased (§factor 1.7) with respect to Sbc-Sc galaxies, when considering the $300 \mathrm{Myr}$ period. 

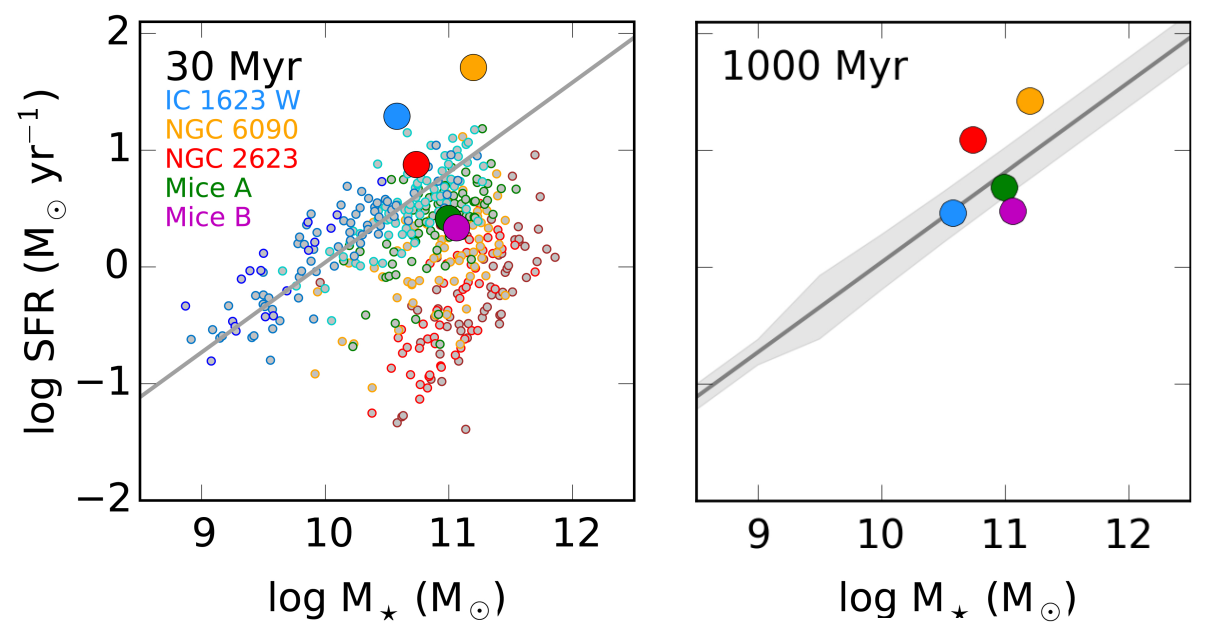

Fig. 7. Global average SFR versus the galaxy stellar mass for the four mergers of our sample at the two different time scales $\left(t_{\mathrm{SF}}=30 \mathrm{Myr}\right.$ (left) and $1 \mathrm{Gyr}($ right)). For comparison, we show the MSSF (grey line) as derived by González Delgado et al. (2016) using late type Sc spirals of CALIFA and using a $t_{\mathrm{SF}}=30 \mathrm{Myr}$. In the left panel, all the galaxies are shown with small dots, colour-coded by Hubble type following González Delgado et al. (2016), with a brown to blue colour palette that represents Hubble types from ellipticals to late spirals. In the right panel the light grey shaded regions indicate the $1 \sigma$ dispersion of the sample. The positions of the Mice, the pre-merger LIRGs IC 1623 W and NGC 6090, and NGC 2623, are shown with solid circles following the same colour coding as in previous figures.

- $t_{\mathrm{SF}}=1 \mathrm{Gyr}:$ The $\operatorname{sSFR}\left(t_{\mathrm{SF}}=1 \mathrm{Gyr}\right)$ in the centre of the mergers differ with respect to $\mathrm{Sbc}(\mathrm{Sc})$ galaxies by $0.55 \mathrm{dex}$ $(0.30 \mathrm{dex})$ in NGC 2623, $0.43 \mathrm{dex}(0.18 \mathrm{dex})$ for IC $1623 \mathrm{~W}$, 0.31 dex $(0.06 \mathrm{dex})$ for NGC $6090,-0.08 \mathrm{dex}(-0.33 \mathrm{dex})$ for Mice A, and $-0.94 \operatorname{dex}(-1.19 \mathrm{dex})$ for Mice B. Analogously, in the outer parts we find differences between our mergers and $\mathrm{Sbc}(\mathrm{Sc})$ galaxies of $0.38 \mathrm{dex}(0.19 \mathrm{dex})$ in NGC 2623, 0.07 dex $(-0.12$ dex $)$ in NGC 6090, -0.36 dex $(-0.55 \mathrm{dex})$ in Mice B, -0.42 dex $(-0.61 \mathrm{dex})$ in IC 1623 $\mathrm{W}$, and $-0.56 \mathrm{dex}(-0.75 \mathrm{dex})$ in Mice A. With respect to the gradient in the inner $1 \mathrm{HLR}$, the $\operatorname{sSFR}\left(t_{\mathrm{SF}}=1 \mathrm{Gyr}\right)$ gradient is from more positive to more negative; $1.60 \mathrm{dex} / \mathrm{HLR}$ in Mice B, 0.55 dex/HLR in Sbc galaxies, 0.54 dex/HLR in Sc galaxies, $0.32 \mathrm{dex} / \mathrm{HLR}$ in NGC 2623, 0.27 dex/HLR in NGC 6090, 0.23 dex/HLR in Mice A, and -0.33 dex/HLR in IC $1623 \mathrm{~W}$. The $\operatorname{sSFR}\left(t_{\mathrm{SF}}=1 \mathrm{Gyr}\right)$ gradient is positive for the control spirals and all the mergers except IC $1623 \mathrm{~W}$. This points to a quenching in the central parts of the galaxies in comparison with the outer parts in the last 1 Gyr. IC 1623 $\mathrm{W}$, however, presents the opposite behaviour.

The sSFR can be interpreted as the inverse of a time scale for the $\mathrm{SF}(\tau)$. These results in the last $30 \mathrm{Myr}$ indicate that $\tau$ is much shorter in LIRG-mergers than in spiral galaxies of similar mass. In particular, this time is $\sim 2.7$ Gyr for IC $1623 \mathrm{~W}, \sim 3.2 \mathrm{Gyr}$ for NGC 6090, 7.0 Gyr for NGC 2623, while for the Mice and control spirals $\tau$ is above the Hubble time (González Delgado et al. 2016). This means that the three LIRG mergers can double their actual stellar mass faster than Sbc-Sc galaxies, if the SFR proceeds at the same rate than in the last $32 \mathrm{Myr}$.

In contrast, for $t_{\mathrm{SF}}=1 \mathrm{Gyr}, \tau$ is quite similar for the earlystage LIRG mergers and the Sbc-Sc galaxies ( 10 Gyr). This reinforces the result that the enhancement of star formation in these objects occurs in time scales of few $100 \mathrm{Myr}$. On the contrary, for the more advanced merger NGC 2623, $\tau(<1$ Gyr) $5 \mathrm{Gyr}$, pointing to a star formation history more extended in time.

\section{Discussion}

\subsection{Global enhancement of the star formation in mergers}

It is well established that star-forming galaxies of the local Universe show a correlation between their recent star formation rate and galaxy stellar mass known as the main sequence of star forming galaxies (MSSF; Brinchmann et al. 2004). The logarithmic slope of this relation is sub-lineal and close to 1 , and the dispersion is small, of 0.2-0.3 dex (Renzini \& Peng 2015). High redshift galaxy surveys have also proved that this relation persists at least to a redshift of approximately four (Peng et al. 2010; Wuyts et al. 2011; Speagle et al. 2014).

Recently, a bimodal distribution in the SFR-M $M_{\star}$ plane is found in $z=4-5$ galaxies; probing the existence of a starburst sequence, with a much more effective star formation mode (i.e. mergers, perturbations) than the secular halo gas accretion occurring in main-sequence star forming galaxies. Previous studies failed in recognising the starburst sequence, as they only focuse in the most massive systems $\left(M_{\star}>10^{10} M_{\odot}\right)$. When low and intermediate mass galaxies are taken into account, the starburst sequence is clearly evident, and contributes to $>50 \%$ of the cosmic SFR density at $z=4-5$ (Caputi et al. 2017).

Local galaxies with recent intense starbursts, as (U)LIRGs, can also be significantly above of the MSSF. In a recent study Guo et al. (2016) found that roughly three quarters of the advanced ULIRGs of their sample are located above $1 \sigma$ of the MSSF. However, this is not always true for LIRGs; as the enhancement of the star formation with respect to the MSSF depends on the time scale and merger phase. For example, Pereira-Santaella et al. (2015) in their sample of local LIRGs, find that the global SFH of these galaxies can be grouped in three categories: 1) almost half of the objects with a recent starburst in the last 10-100 Myr; 2) a similar fraction of galaxies with constant SFR; and 3) only a few objects with a declining SFR. In the first group, all of them are significantly above the MSSF in time scales of less than $30 \mathrm{Myr}$; but at $1 \mathrm{Gyr}$, the objects are on the MSSF or below. Most of the LIRGs that are above the MSSF are mergers and have a high $s S F R>0.8 \mathrm{Gyr}^{-1}$.

Figure 7 shows the global average SFR vs galaxy stellar mass for the four mergers of our sample at $t_{\mathrm{SF}}=30 \mathrm{Myr}$ and 1 Gyr time scales. For comparison, the MSSF as derived by González Delgado et al. (2016) using late type Sc spirals of CALIFA and $t_{\mathrm{SF}}=30 \mathrm{Myr}$ is drawn as a greyline. With the exception of the Mice, the global SFR in the other systems are enhanced with respect to the MSSF galaxies, in the $t_{\mathrm{SF}} \leq 30 \mathrm{Myr}$ time scale. In particular, the SFR is enhanced by a 
factor of approximately six in IC $1623 \mathrm{~W}$, approximately six in NGC 6090, and approximately two in NGC 2623. The Mice are seen after first passage (Wild et al. 2014), and it is probably the least advanced merger, so the star formation is still not enhanced. This result was expected given that the Mice have FIR luminosities below $10^{11} L_{\odot}$, the limit to be classified as a LIRG.

It is also interesting that although the LIRG-mergers are above the MSSF at $t_{\mathrm{SF}} \leq 30 \mathrm{Myr}$, at $t_{\mathrm{SF}}=1 \mathrm{Gyr}$ IC $1623 \mathrm{~W}$ is on the MSSF, while in NGC 6090 the SFR is enhanced by a factor of approximately three with respect to Sc MSSF galaxies, but is half the one measured at $t_{\mathrm{SF}} \leq 30 \mathrm{Myr}$. On the contrary, in NGC 2623 there is a slight increase of the SFR in this longest time scale, which is a factor of approximately three larger than in Sc MSSF galaxies. This is expected given the known presence and relevance of intermediate-age stellar populations in this system (Cortijo-Ferrero et al. 2017b).

This points out that a major phase of star formation in the merger LIRGs occurs in time scales of $10^{7} \mathrm{yr}$ to few $10^{8} \mathrm{yr}$. Our results are more in agreement with Rodríguez Zaurín et al. (2009, 2010) where the optical light of local ULIRGs (mostly mergers in different evolutionary stages) have important contribution of stellar populations of 300-500 Myr. They also agree with Pereira-Santaella et al. (2015) where the duration of the bursts is on time scales of $\leq 10$ to few $100 \mathrm{Myr}$ for the LIRGs that show merger signatures. Star formation time scales of 40260 Myr have been also found by Marcillac et al. (2006) in their LIRGs at redshift $z \sim 0.7$, but none of their galaxies show time scales of less than a few $10 \mathrm{Myr}$ as we detect in LIRG-mergers. In the more advanced merger NGC 2623 an even more intense phase of star formation occurred in a longer time scale of $\sim 1$ Gyr. These results are in agreement with those of Alonso-Herrero et al. (2010) that studied a small sample of LIRGs, and found that the dominant stellar population is at least 1-2 Gyr old. However, we note that most of their galaxies are isolated or weakly interacting local LIRGs.

\subsection{The extent and relevance of the different phases of star formation}

It is interesting now to discuss the relevance of the different phases of enhancement of the star formation and their spatial extent. Using the specific SFR intensity as a mass-independent SFR tracer, what we have found is:

$-t_{\mathrm{SF}}=30$ Myr: In the early-stage merger LIRG IC $1623 \mathrm{~W}$ the star formation in the last $30 \mathrm{Myr}$ is enhanced with respect to the control spirals both in the central regions (by a factor of seven) and in the disk (by a factor of five), but in the early-stage merger NGC 6090 the enhancement is important in the central region (by a factor of seven) while in the disk it is much more moderate (a factor of two) in comparison to control spirals. For the merger NGC 2623, the young star formation in the last $30 \mathrm{Myr}$ is enhanced in the central region (by a factor of three), while at the outer parts it is similar to the control spirals. On the contrary, in Mice A, the young star formation in the last $30 \mathrm{Myr}$ is similar to the control spirals in the centre, but decreased by a factor of eleven in the disk, and in Mice B it is decreased both in the centre (by a factor of 14), and in the disk (by a factor of four).

The results are qualitatively the same when considering a longer young-intermediate time scale of $\lesssim 300 \mathrm{Myr}$, with some differences only in the numbers.

$-t_{\mathrm{SF}}=1$ Gyr: However, the star formation in the last Gyr is only enhanced (by a factor of three) in the central regions of NGC 2623, with respect to control spirals, while in the pre-mergers the enhancement is very low (by factors of less than two). In the centre of Mice A it is comparable to the control spirals, and in Mice B it is decreased by a factor of eleven. In the outer regions, the star formation in the last Gyr is enhanced with respect to the control spirals by a factor of two in NGC 2623, while for the pre-merger LIRGs it is comparable to the control spirals (NGC 6090), or a factor of three to four lower (IC $1623 \mathrm{~W}$, Mice A and B), as if the star formation has been inhibited in this time period.

We can also quantify the relative impact of these different periods of star formation by comparing the amount of mass formed in each period. Using the mass fractions reported in Table 2 we obtain very similar numbers compared to those previously reported, and therefore, reaching totally consistent conclusions when considering the amounts of mass formed relative to the non-interacting spirals. Basically, the two pre-merger LIRGs formed three to seven times more mass, in the last $30 \mathrm{Myr}$, than the non-interacting control spirals, both in the centres, and in the disks, while the merger NGC 2623 formed four times more mass in its centre than Sbc-Sc spirals, but comparable in the disk. In the $300 \mathrm{Myr}$ time scale, IC $1623 \mathrm{~W}$ is the system which formed more mass in comparison to the control spirals, by a factor $\sim 21$ in the centre, and four in the disks. The two other LIRGs, NGC 6090 and NGC 2623, formed significantly more mass than the spirals in their centres (a factor of between five and six), and less in their disk (but still a factor of approximately two more). In the 1 Gyr time scale, only NGC 2623 formed significantly more mass than the non-interacting spirals, by a factor of three in the centre, and two in the disk.

Hence, we conclude that the recent star formation in the three LIRG mergers is enhanced with respect to the Sbc-Sc galaxies. However, the amount and the spatial extension of the enhancement of the star formation depends on the time scale and the galaxy. Thus, on short $\left(t_{\mathrm{SF}}=30 \mathrm{Myr}\right)$ time scales the enhancement is quite significant in the three systems, but on longer time scales $\left(t_{\mathrm{SF}}=1 \mathrm{Gyr}\right)$ it is only relevant in the advanced merger NGC 2623. In the Mice, the star formation is mostly inhibited with respect to the control spirals, specially in Mice B. We think this is due to a lack of gas in this progenitor, because it has a significantly lower gas mass fraction $(\sim 6 \%)$ in comparison to the Mice A $(\sim 13 \%)$ (Wild et al. 2014), and typical Sbc (Sc) spirals $\sim 13$ (16)\% (Rubin et al. 1985). This gas deficiency could be caused by negative feedback from the AGN which is present in the centre of Mice B (González-Martín et al. 2009; Haan et al. 2011; Masegosa et al. 2011), as Mice B has a total stellar mass of $\sim 1.5 \times 10^{11} M_{\odot}$, compatible with the regime where feedback is dominated by AGN outflows (Shankar et al. 2006).

\subsection{Evolutionary scheme}

High spatial resolution hydrodynamic simulations of disk galaxies mergers predict that extended starbursts arise spontaneously after the first pericentre passage, due to fragmentation of the gas clouds produced by the increase of the supersonic turbulence of ISM. According to these models, a merger-induced nuclear starburst is also present, but it occurs later in the merger sequence, after the second pericentre passage (Teyssier et al. 2010; Powell et al. 2013).

Although a systematic spatially resolved analysis of a large and complete observational sample of mergers is necessary to properly test the validity of these simulations and attain more general conclusions, our sample provides some clues from the 
observational point of view. The most advanced merger in our sample, NGC 2623, which has already passed coalescence, seems to reproduce the predictions from simulations, showing both an extended SFR enhancement about $\lesssim 1$ Gyr ago, relic of the first pericentre passage epoch, and a current SFR enhancement in the last $30 \mathrm{Myr}$, located in the central 0.5 HLR but not in the outer parts, consistent with the second pericentre passage and final coalescence. This is consistent with what we already found through the stellar population analysis in this system (Cortijo-Ferrero et al. 2017b). We note that advanced postcoalescence systems are ideal places where to apply the fossil method to unveil past star formation epochs, as both the extended and nuclear starbursts should be identifiable in the spectra of most of these systems if the current merger scenario is correct.

Analogously, in the two pre-merger LIRGs IC $1623 \mathrm{~W}$ and NGC 6090, which are somewhere located between first pericentre passage and prior to coalescence, the most remarkable increase of the SFR with respect to non-interacting spirals occurred in the last $30 \mathrm{Myr}$, and is spatially extended, in agreement with current simulations. We note that IC $1623 \mathrm{~W}$ could represent a more evolved state as the extended SFR enhancement was already traced by $\sim 300 \mathrm{Myr}$ populations. This is in agreement to what we found in Cortijo-Ferrero et al. (2017a). The $\sim 1 \mathrm{Gyr}$ populations do not show significantly enhanced mass and SFR contributions with respect to non-interacting spirals in neither the outer parts nor the centres.

Contrary to what was expected, although Mice passed the first pericentre passage about $170 \mathrm{Myr}$ ago (Barnes 2004), where a starburst should have occurred, there exists no evidence of an enhanced SFR in the last $30 \mathrm{Myr}$ or $300 \mathrm{Myr}$ in this system: on the contrary, the star formation is mainly inhibited. The Mice are probably the least evolved merger of the sample, and the fact that the gas fraction of Mice B is smaller than in most non-interacting spirals shows that there are many factors that determine when, where, and with which intensity the starbursts will occur. Also, the Mice are close to a prograde orbit (Barnes 2004), while simulations by Di Matteo et al. (2007) show that retrograde encounters (when the galaxies spin is antiparallel) have larger star formation efficiencies, even if prograde encounters develop more pronounced asymmetries than retrograde ones, whose remnants are more compact.

These simulations show us that the merger time scale, SF enhancement, and remnant properties of galaxy mergers, depend on several factors such as: the morphologies of the progenitors, the availability of gas (gas-rich or gas-poor), and the orbital characteristics. It is therefore necessary to make a detailed spatially resolved study of mergers covering all the merger stages, with information about the total gas content and the kinematics, to shed more light on the merger evolutionary sequence.

\section{Summary and conclusions}

Using IFS data from the CALIFA survey and PMAS in LArr mode, we have analyzed the spatially resolved star formation history of a small sample of local mergers: the early-stage mergers (Mice, IC 1623, and NGC 6090) and a more advanced merger, NGC 2623, to determine if there is and quantify an enhancement of the star formation and trace its time scale and spatial extent. A full spectral fitting analysis was performed using the STARLIGHT code and a combination of the single stellar populations models by Vazdekis et al. (2010) and González Delgado et al. (2005), using 156 spectra of 39 different ages from $1 \mathrm{Myr}$ to $14 \mathrm{Gyr}$, and four metallicities $(Z=0.2,0.4$, 1 , and $1.6 Z_{\odot}$ ). The spectral fitting results are processed through
PycAsso pipeline to derive the $2 \mathrm{D}(R \times t)$ maps, from which we obtain the spatially resolved star formation rate (SFR), specific SSFR, and the intensity of the SFR $\left(\Sigma_{\mathrm{SFR}}\right)$, over three different time scales (30 Myr, $300 \mathrm{Myr}$, and $1 \mathrm{Gyr}$ ).

Our main results are:

1. A major phase of star formation in the merger LIRGs is occurring in time scales of $10^{7} \mathrm{yr}$ to few $10^{8} \mathrm{yr}$, with global SFR enhancements of between approximately two and six with respect to the MSSF galaxies. In the more advanced merger NGC 2623 a previous phase of star formation occurred in a longer time scale of $\sim 1$ Gyr.

2. From our spatially resolved analysis, we have quantified the extension and relative impact of the different periods of star formation in the last 30, 300, and 1000 Myr. Using the SSFR as a mass-independent SFR tracer, we find that the two pre-merger LIRGs present a sSFR enhancement in the last $30 \mathrm{Myr}$ of two to seven times that in non-interacting control spirals, both in the centres, and in the disks, while in the merger NGC 2623 the enhancement is a factor of three in the centre, but comparable to Sbc-Sc galaxies in the outer regions. In the $300 \mathrm{Myr}$ time scale, IC $1623 \mathrm{~W}$ is the system which presents a greater sSFR enhancement in comparison to the control spirals. Finally, in the $1 \mathrm{Gyr}$ time scale, only NGC 2623 formed significantly more mass than the noninteracting spirals, by a factor of three in the centre, and two in the disk.

3. The spatially resolved study of the three LIRG-mergers reveals that their SFH is consistent with the predictions from high spatial resolution simulations. Extended starbursts arise after the first pericentre passage (consistent with the enhancement of the extended SF in the last $\$ 30-300 \mathrm{Myr}$ in IC $1623 \mathrm{~W}$ and NGC 6090, and $\lesssim 1$ Gyr in NGC 2623), while a nuclear starburst occurs later in more advanced mergers, after the second pericentre passage and/or coalescence (i.e. as reflected by the enhancement of the central SF in NGC 2623 in the last $30 \mathrm{Myr}$ ). A systematic analysis of a larger and more complete observational sample of mergers is necessary to further test the validity of these simulations.

4. The Mice are the only system not showing a significant enhancement of the spatially extended star formation, but rather it is inhibited, that is more severe in Mice B. The Mice are not classified as LIRG, and it is probably the least advanced merger in our sample. The fact that the gas fraction of Mice B is smaller than in most non-interacting spirals, and that the Mice are close to a prograde orbit, presents a new challenge for the models, which must cover a larger space of parameters in terms of the availability of gas and the orbital characteristics.

Acknowledgements. CALIFA is the first legacy survey carried out at Calar Alto. The CALIFA collaboration would like to thank the IAA-CSIC and MPIA-MPG as major partners of the observatory, and CAHA itself, for the unique access to telescope time and support in manpower and infrastructures. We also thank the CAHA staff for the dedication to this project. Support from the Spanish Ministerio de Economía y Competitividad, through projects AYA2016-77846-P, AYA2014-57490-P, AYA2010-15081, and Junta de Andalucía FQ1580, AYA2010-22111-C03-03, AYA2010-10904E, AYA201342227P, RyC-2011-09461, AYA2013-47742-C4-3-P, EU SELGIFS exchange programme FP7-PEOPLE-2013-IRSES-612701, and CONACYT-125180 and DGAPA-IA100815. We also thank the Viabilidad, Diseño, Acceso y Mejora funding program, ICTS-2009-10, for funding the data acquisition of this project. A.L.d.A., E.A.D.L. and R.C.F. thank the hospitality of the IAA and the support of CAPES and CNPq. R.G.D. acknowledges the support of CNPq (Brazil) through Programa Ciência sem Fronteiras (401452/2012-3). We thank the support of the IAA Computing group. This research made use of Python (http:// www . python. org); Numpy (Van Der Walt et al. 2011), and Matplotlib (Hunter 2007). 


\section{References}

Alonso-Herrero, A., García-Marín, M., Rodríguez Zaurín, J., et al. 2010, A\&A, 522, A7

Asari, N. V., Cid Fernandes, R., Stasińska, G., et al. 2007, MNRAS, 381, 263

Barnes, J. E. 2004, MNRAS, 350, 798

Barrera-Ballesteros, J. K., Sánchez, S. F., García-Lorenzo, B., et al. 2015, A\&A 579, A45

Bica, E. 1988, A\&A, 195, 76

Brinchmann, J., Charlot, S., White, S. D. M., et al. 2004, MNRAS, 351, 115

Bruzual, G., \& Charlot, S. 2003, MNRAS, 344, 1000

Calzetti, D., Armus, L., Bohlin, R. C., et al. 2000, ApJ, 533, 682

Caputi, K. I., Deshmukh, S., Ashby, M. L. N., et al. 2017, ApJ, 849, 45

Cappellari, M., \& Copin, Y. 2003, MNRAS, 342, 345

Cardelli, J. A., Clayton, G. C., \& Mathis, J. S. 1989, ApJ, 345, 245

Cid Fernandes, R., Leão, J. R. S., \& Lacerda, R. R. 2003, MNRAS, 340, 29

Cid Fernandes, R., Mateus, A., Sodré, L., Stasińska, G., \& Gomes, J. M. 2005 MNRAS, 358, 363

Cid Fernandes, R., Pérez, E., García Benito, R., et al. 2013, A\&A, 557, A86

Cid Fernandes, R., González Delgado, R. M., García Benito, R., et al. 2014, A\&A, 561, A130

Cortijo-Ferrero, C., González Delgado, R. M., Pérez, E., et al. 2017a, MNRAS 467,3898

Cortijo-Ferrero, C., González Delgado, R. M., Pérez, E., et al. 2017b, A\&A, 606, A95

Dekel, A., Birnboim, Y., Engel, G., et al. 2009, Nature, 457, 451

Di Matteo, P., Combes, F., Melchior, A.-L., \& Semelin, B. 2007, A\&A, 468, 61

Di Matteo, P., Bournaud, F., Martig, M., et al. 2008, A\&A, 492, 31

Elmegreen, D. M., Elmegreen, B. G., Kaufman, M., et al. 2006, ApJ, 642, 158

Evans, A. S., Vavilkin, T., Pizagno, J., et al. 2008, ApJ, 675, L69

Fensch, J., Renaud, F., Bournaud, F., et al. 2017, MNRAS, 465, 1934

García-Benito, R., Zibetti, S., Sánchez, S. F., et al. 2015, RA\&A, 576, A135

García-Benito, R., et al., 2017, A\&A, submitted

González Delgado, R. M., Cid Fernandes, R., Pérez, E., et al. 2004, ApJ, 605, 127

González Delgado, R. M., Cerviño, M., Martins, L. P., Leitherer, C., \& Hauschildt, P. H. 2005, MNRAS, 357, 945

González-Martín, O., Masegosa, J., Márquez, I., Guainazzi, M., \& JiménezBailón, E. 2009, A\&A, 506, 1107

González Delgado, R. M., Pérez, E., Cid Fernandes, R., et al. 2014, A\&A, 562, A47

González Delgado, R. M., García-Benito, R., Pérez, E., et al. 2015, A\&A, 581, A103

González Delgado, R. M., Cid Fernandes, R., Pérez, E., et al. 2016, A\&A, 590, A44

González Delgado, R. M., Pérez, E., Cid Fernandes, R., et al. 2017, A\&A, submitted

Guo, R., Hao, C.-N., Xia, X. Y., Mao, S., \& Shi, Y. 2016, ApJ, 826, 30

Haan, S., Armus, L., Laine, S., et al. 2011, ApJS, 197, 27

Hernquist, L. 1989, Nature, 340, 687

Hopkins, P. F., Cox, T. J., Hernquist, L., et al. 2013, MNRAS, 430, 1901

Hunter, J. D. 2007, Comput. Sci. Eng., 9, 90

Husemann, B., Jahnke, K., Sánchez, S. F., et al. 2013, A\&A, 549, A87

Kaviraj, S. 2014, MNRAS, 437, L41

Kelz, A., Verheijen, M. A. W., Roth, M. M., et al. 2006, PASP, 118, 129

Kennicutt, Jr., R. C. 1998, ARA\&A, 36, 189
Kim, D.-C., Evans, A. S., Vavilkin, T., et al. 2013, ApJ, 768, 102

Knapen, J. H., Cisternas, M., \& Querejeta, M. 2015, MNRAS, 454, 1742

Lofthouse, E. K., Kaviraj, S., Conselice, C. J., Mortlock, A., \& Hartley, W. 2017, MNRAS, 465, 2895

Maraston, C., Pforr, J., Renzini, A., et al. 2010, MNRAS, 407, 830

Maraston, C., Pforr, J., Henriques, B. M., et al. 2013, MNRAS, 435, 2764

Marcillac, D., Elbaz, D., Charlot, S., et al. 2006, A\&A, 458, 369

Masegosa, J., Márquez, I., Ramirez, A., \& González-Martín, O. 2011, A\&A, $527, \mathrm{~A} 23$

Mihos, J. C., \& Hernquist, L. 1994, ApJ, 425, L13

Mihos, J. C., \& Hernquist, L. 1996, ApJ, 464, 641

Moreno, J., Torrey, P., Ellison, S. L., et al. 2015, MNRAS, 448, 1107

Peng, Y.-J., Lilly, S. J., Kovač, K., et al. 2010, ApJ, 721, 193

Pereira-Santaella, M., Alonso-Herrero, A., Colina, L., et al. 2015, A\&A, 577, A78

Pérez, E., Cid Fernandes, R., González Delgado, R. M., et al. 2013, ApJ, 764, L1

Powell, L. C., Bournaud, F., Chapon, D., \& Teyssier, R. 2013, MNRAS, 434, 1028

Renaud, F., Bournaud, F., \& Duc, P.-A. 2015, MNRAS, 446, 2038

Renaud, F., Famaey, B., \& Kroupa, P. 2016, MNRAS, 463, 3637

Renzini, A., \& Peng, Y.-J. 2015, ApJ, 801, L29

Rodighiero, G., Daddi, E., Baronchelli, I., et al. 2011, ApJ, 739, L40

Rodríguez Zaurín, J., Tadhunter, C. N., \& González Delgado, R. M. 2009 MNRAS, 400, 1139

Rodríguez Zaurín, J., Tadhunter, C. N., \& González Delgado, R. M. 2010, MNRAS, 403, 1317

Roth, M. M., Kelz, A., Fechner, T., et al. 2005, PASP, 117, 620

Rubin, V. C., Burstein, D., Ford, Jr., W. K., \& Thonnard, N. 1985, ApJ, 289, 81

Sánchez, S. F., Kennicutt, R. C., Gil de Paz, A., et al. 2012, A\&A, 538, A8

Sánchez, S. F., Pérez, E., Sánchez-Blázquez, P., et al. 2016, Rev. Mex. Astron. Astrofis., 52, 171

Sanders, D. B., \& Mirabel, I. F. 1996, ARA\&A, 34, 749

Sanders, D. B., Mazzarella, J. M., Kim, D.-C., Surace, J. A., \& Soifer, B. T. 2003, AJ, 126, 1607

Shankar, F., Lapi, A., Salucci, P., De Zotti, G., \& Danese, L. 2006, ApJ, 643, 14

Smith, B. J., Zaragoza-Cardiel, J., Struck, C., Olmsted, S., \& Jones, K. 2016, AJ, 151,63

Speagle, J. S., Steinhardt, C. L., Capak, P. L., \& Silverman, J. D. 2014, ApJS, 214,15

Starkenburg, T. K., Helmi, A., \& Sales, L. V. 2016, A\&A, 587, A24

Surace, J. A., Sanders, D. B., Vacca, W. D., Veilleux, S., \& Mazzarella, J. M. 1998, ApJ, 492, 116

Surace, J. A., Sanders, D. B., \& Evans, A. S. 2000, ApJ, 529, 170

Tacchella, S., Carollo, C. M., Renzini, A., et al. 2015, Science, 348, 314

Teyssier, R., Chapon, D., \& Bournaud, F. 2010, ApJ, 720, L149

Van Der Walt, S., Colbert, S. C., \& Varoquaux, G. 2011, Comput. Sci. Eng., 13,

Vazdekis, A., Sánchez-Blázquez, P., Falcón-Barroso, J., et al. 2010, MNRAS, 404, 1639

Veilleux, S., Kim, D.-C., \& Sanders, D. B. 2002, ApJS, 143, 315

Verheijen, M. A. W., Bershady, M. A., Andersen, D. R., et al. 2004, Astron. Nachr., 325, 151

Wang, Z., Fazio, G. G., Ashby, M. L. N., et al. 2004, ApJS, 154, 193

Wild, V., Rosales-Ortega, F., Falcón-Barroso, J., et al. 2014, A\&A, 567, A132

Wilson, C. D., Harris, W. E., Longden, R., \& Scoville, N. Z. 2006, ApJ, 641, 763

Wolf, C., Bell, E. F., McIntosh, D. H., et al. 2005, ApJ, 630, 771

Wuyts, S., Förster Schreiber, N. M., van der Wel, A., et al. 2011, ApJ, 742, 96 